\title{
Root Locus Practical Sketching Rules for Fractional-Order Systems
}

\author{
António M. Lopes ${ }^{1}$ and J. A. Tenreiro Machado ${ }^{2}$ \\ ${ }^{1}$ Institute of Mechanical Engineering, UISPA, Faculty of Engineering, University of Porto, \\ Rua Dr. Roberto Frias, 4200-465 Porto, Portugal \\ ${ }^{2}$ Institute of Engineering, Polytechnic of Porto, Rua Dr. António Bernardino de Almeida 431, 4200-072 Porto, Portugal
}

Correspondence should be addressed to J. A. Tenreiro Machado; jtm@isep.ipp.pt

Received 26 April 2013; Accepted 2 June 2013

Academic Editor: Dumitru Baleanu

Copyright (C) 2013 A. M. Lopes and J. A. Tenreiro Machado. This is an open access article distributed under the Creative Commons Attribution License, which permits unrestricted use, distribution, and reproduction in any medium, provided the original work is properly cited.

For integer-order systems, there are well-known practical rules for RL sketching. Nevertheless, these rules cannot be directly applied to fractional-order (FO) systems. Besides, the existing literature on this topic is scarce and exclusively focused on commensurate systems, usually expressed as the ratio of two noninteger polynomials. The practical rules derived for those do not apply to other symbolic expressions, namely, to transfer functions expressed as the ratio of FO zeros and poles. However, this is an important case as it is an extension of the classical integer-order problem usually addressed by control engineers. Extending the RL practical sketching rules to such FO systems will contribute to decrease the lack of intuition about the corresponding system dynamics. This paper generalises several RL practical sketching rules to transfer functions specified as the ratio of FO zeros and poles. The subject is presented in a didactic perspective, being the rules applied to several examples.

\section{Introduction}

Root locus (RL) analysis is a graphical method that shows how the poles of a closed-loop transfer function change with relation to a given system parameter $[1,2]$. Usually, the chosen parameter is a proportional gain, $K \geq 0$, included in a unity feedback closed-loop controlled system (Figure 1).

The open and closed-loop transfer functions are given by $G_{\mathrm{OL}}(s)=K \cdot G(s)$ and $G_{\mathrm{CL}}(s)=K \cdot G(s) /[1+K \cdot G(s)]$, respectively. The denominator of $G_{\mathrm{CL}}(s)$ is the characteristic equation, and its roots are the system closed-loop poles. Every point of the RL simultaneously satisfies the well-known argument (angle) and magnitude conditions given by

$$
\begin{aligned}
\arg \{K \cdot G(s)\}= & (2 h+1) \cdot 180^{\circ}, \quad h=0, \pm 1, \pm 2, \ldots, \\
& \operatorname{abs}\{K \cdot G(s)\}=1 .
\end{aligned}
$$

The RL is a classical and powerful tool for the dynamical analysis and design of integer-order linear time-invariant (LTI) systems [1-6]. Nowadays, there are efficient numerical algorithms, implemented in several software packages (e.g., MATLAB, Octave, Scilab, and FreeMat) [7-10] that take advantage of the powerful digital processors of modern computers to perform RL analysis. For fractional-order (FO) systems, while several studies addressing RL are available [1117], the problem is more difficult and researchers have mainly preferred to adopt frequency-based methods.

On the other hand, the ability to quickly sketch RL by hand is invaluable in making fundamental decisions early in the design process. For integer-order systems, there are well-known practical rules for RL sketching, but those cannot be directly applied to FO systems. Moreover, the existing literature on this topic exclusively focuses on the particular case of commensurate FO systems that occur when truncating real valued integro-differential orders up to a finite precision $[15,16]$. This allows the generalisation of some rules to FO systems, but limiting the precision and the type of symbolic expressions $[17,18]$. The rules for commensurate FO systems do not apply to transfer functions expressed as the ratio of FO zeros and poles. However, this is an important 


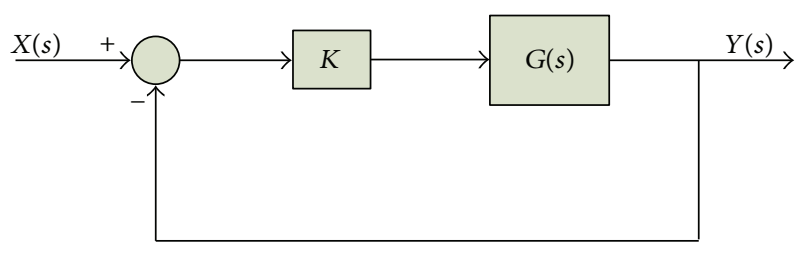

FIGURE 1: Unity feedback closed-loop controlled system.

case as it is an extension of the classical integer-order problem usually addressed by control engineers when dealing with RL analysis.

In this paper, we extend several practical rules, available to sketch the RL of integer-order systems, to the FO domain. The main contribution is that the practical sketching rules apply to open-loop transfer functions expressed as the ratio of FO zeros and poles, contributing to fill the gap in the existing literature about this topic. The subject is presented in a didactic perspective, being the rules applied to several examples that contribute to reduce the lack of intuition about the corresponding system dynamics.

Bearing these ideas in mind, the paper is organized as follows. Section 2 introduces fundamental concepts related to fractional calculus. Section 3 analyses several FO systems and generalises the RL rules to a class of FO systems. Finally, Section 4 draws the main conclusions.

\section{Fractional Calculus}

Fractional calculus (FC) denotes the branch of calculus that extends the concepts of integrals and derivatives to noninteger and complex orders [19-23]. During the last years, FC was found to play a fundamental role in the modelling of a considerable number of phenomena [24-29] and emerged as an important tool for the study of dynamical systems where classical methods reveal strong limitations. Nowadays, the application of FC concepts includes a wide spectrum of studies [30-33], going from the dynamics of financial markets $[34,35]$, biological systems $[36,37]$, earth sciences [38], and DNA sequencing [39] up to mechanical [40-43], electrical [44-46], and control systems [21, 24].

The generalisation of the concept of derivative and integral to noninteger orders, $\alpha$, has been addressed by several mathematicians. The Riemann-Liouville, GrünwaldLetnikov, and Caputo definitions of fractional derivative are the most used and are given, respectively, by [47]

$$
\begin{gathered}
{ }_{a}^{\mathrm{RL}} D_{t}^{\alpha} f(t)=\frac{1}{\Gamma(n-\alpha)} \frac{d^{n}}{d t^{n}} \int_{a}^{t} \frac{f(\tau)}{(t-\tau)^{\alpha-n+1}} d \tau, \\
n-1<\alpha<n, \\
{ }_{a}^{\mathrm{GL}} D_{t}^{\alpha} f(t)=\lim _{h \rightarrow 0} \frac{1}{h^{\alpha}} \sum_{k=0}^{[(t-a) / h]}(-1)^{k}\left(\begin{array}{l}
\alpha \\
k
\end{array}\right) f(t-k h),
\end{gathered}
$$

$$
\begin{array}{r}
{ }_{a}^{C} D_{t}^{\alpha} f(t)=\frac{1}{\Gamma(n-\alpha)} \int_{a}^{t} \frac{f^{(n)}(\tau)}{(t-\tau)^{\alpha-n+1}} d \tau, \\
n-1<\alpha<n,
\end{array}
$$

where $\Gamma(\cdot)$ represents Euler's gamma function, $[x]$ is the integer part of $x$, and $h$ is a time step.

The Laplace transform applied to (2) yields

$$
L\left\{{ }_{a}^{\mathrm{RL}} D_{t}^{\alpha} f(t)\right\}=s^{\alpha} L\{f(t)\}-\sum_{k=0}^{n-1} s_{0}^{k \mathrm{RL}} D_{t}^{\alpha-k-1} f\left(0^{+}\right),
$$

where $L$ and $s$ denote the Laplace operator and variable, respectively, and $t$ represents time.

The general LTI, single-input-single-output (SISO), and FO incommensurate system can be represented by [48]

$$
\begin{aligned}
& a_{n} D^{\alpha_{n}} y(t)+a_{n-1} D^{\alpha_{n-1}} y(t)+\cdots+a_{0} D^{\alpha_{0}} y(t) \\
& \quad=b_{m} D^{\beta_{m}} x(t)+b_{m-1} D^{\beta_{m-1}} x(t)+\cdots+b_{0} D^{\beta_{0}} x(t),
\end{aligned}
$$

where $x(t)$ and $y(t)$ represent the system input and output, respectively, $D^{(\cdot)}$ is the derivative operator, $\left\{a_{p}, b_{q}\right\} \in$ $\mathbf{R},\left\{\alpha_{p}, \beta_{q}\right\} \in \mathbf{R}_{0}^{+}, p=0, \ldots, n$, and $q=0, \ldots, m$. Besides, it is considered that $\alpha_{n}>\alpha_{n-1}>\cdots>\alpha_{0}, \beta_{m}>\beta_{m-1}>\cdots>$ $\beta_{0}, \alpha_{n}>\beta_{m}$, and $a_{n} \neq 0$.

In the Laplace domain, (6) results in a transfer function given by the ratio of two non-integer polynomials:

$$
G(s)=\frac{b_{m} s^{\beta_{m}}+b_{n-1} s^{\beta_{m-1}}+\cdots+b_{0} s^{\beta_{0}}}{a_{n} s^{\alpha_{n}}+a_{n-1} s^{\alpha_{n-1}}+\cdots+a_{0} s^{\alpha_{0}}} .
$$

If $\alpha_{p}=k_{p} / v, \beta_{q}=k_{q} / v$, with $v \in \mathbf{R}^{+}$and $k_{p}, k_{q} \in \mathbf{N}_{0}$, then (7) is a commensurate FO system and can be written as

$$
G(s)=\frac{\sum_{q=0}^{m} b_{q}\left(s^{1 / \nu}\right)^{k_{q}}}{\sum_{p=0}^{n} a_{p}\left(s^{1 / \nu}\right)^{k_{p}}} .
$$

The FO system is said to be rational if $v \in \mathbf{N}$.

In general, a polynomial $P\left(s^{\alpha}\right)$ is a multivalued function, the domain of which is a Riemann surface with an infinite number of sheets [48]. Only in the particular case of $\alpha$ being rational, the number of sheets will be finite. Such type of function becomes single-valued when an appropriate cut of the complex plane is assumed. This branch cut is not unique, but the negative real axis is usually chosen. In this case, the origin of the complex plane is a branch point and the first Riemann sheet, $\varnothing$, is defined as

$$
\wp=\left\{r e^{j \phi} r \in \mathrm{R}^{+},-\pi<\phi<\pi\right\} .
$$

For example, Figure 2 depicts two Riemann surfaces corresponding to the function $P\left(s^{\alpha}\right)=s^{\alpha}+b(\alpha>0, b>0)$, the roots of which are

$$
s=b^{1 / \alpha} \cdot e^{j(\pi+2 h \pi) / \alpha}, \quad h=0, \pm 1, \pm 2, \ldots ; j=\sqrt{-1} .
$$

For $\alpha=1 / 2$ and $b=1$, the Riemann surface has two sheets (Figure 2(a)), and for $\alpha=4 / 3$ and $b=1$, the 


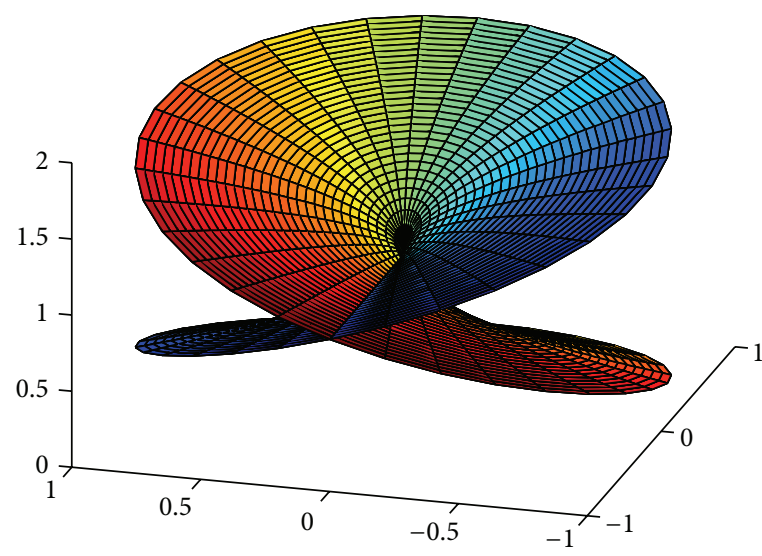

(a)

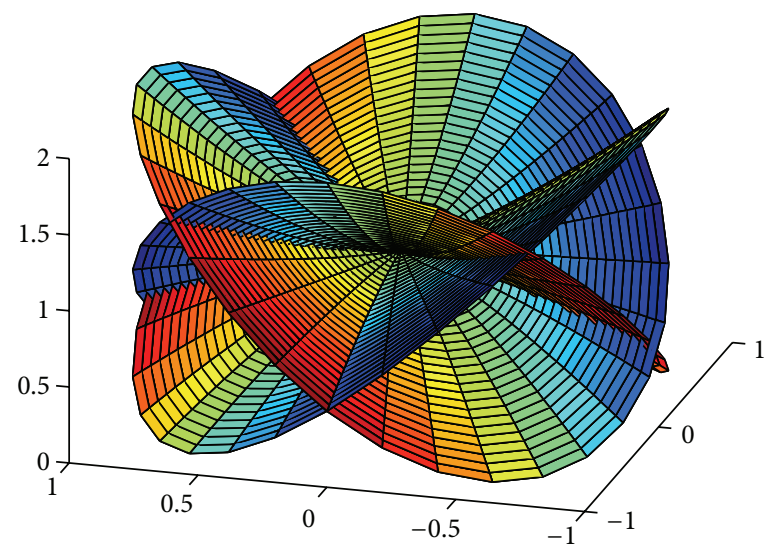

(b)

FIgURE 2: Riemann surfaces: (a) $P\left(s^{\alpha}\right)=s^{1 / 2}+1$ has two sheets; (b) $P\left(s^{\alpha}\right)=s^{4 / 3}+1$ has three sheets.

Riemann surface presents three different sheets (Figure 2(b)). In the former case, there are no roots, and in the latter case, two roots appear on the first sheet. Riemann surfaces are important when dealing with RL of FO systems, as will be seen in Section 3.

\section{Root Locus}

In this section, we assume that the system open-loop transfer function is given by the following:

$$
G(s)=K \frac{\prod_{q=1}^{m}\left(s+b_{q}\right)^{\beta_{q}}}{\prod_{p=1}^{n}\left(s+a_{p}\right)^{\alpha_{p}}},
$$

where $a_{p}, b_{q} \in \mathbf{C}$ and $\alpha_{p}, \beta_{q} \in \mathbf{R}^{+}$.

Equation (11) represents a direct extension to the FO domain of the classical integer-order problem usually addressed by control engineers when dealing with RL analysis. Rules for RL sketching applicable to this case are summarised in Table 1. Only the first Riemann sheet will be considered.

In the sequel, several examples are presented, namely, (i) one FO real pole; (ii) two FO real poles; (iii) one FO pole and one FO zero; (iv) a pair of FO complex conjugate poles. The RL plots are generated using the numeric algorithm presented in [17]. The application of the practical sketching rules is detailed for a few examples, and for all cases, the RL plots serve the purpose of elucidating system dynamics. This will help readers to gain intuition about system behaviour as a function of poles and zeros fractional orders.

3.1. One Fractional-Order Real Pole. In this case, the openloop transfer function is given by

$$
G_{1}(s)=\frac{K}{\left(s+a_{1}\right)^{\alpha_{1}}},
$$

where the RL corresponds to the roots of the characteristic equation

$$
\begin{gathered}
\left(s+a_{1}\right)^{\alpha_{1}}+K=0, \\
s=-a_{1}+K^{1 / \alpha_{1}} \cdot e^{j(2 h+1) \cdot \pi / \alpha_{1}}, \quad h=0, \pm 1, \pm 2, \ldots
\end{gathered}
$$

In general, the RL spreads along several Riemann sheets, meaning that RL branches can begin in one sheet, cross the branch cut, and enter in another sheet. For example, considering $G_{1}(s)$ with $a_{1}=2$ and $\alpha_{1}=1.5$, we verify that the characteristic (13) has roots in two Riemann sheets (Figure 3(a)). However, choosing $\alpha_{1}=1.6$ results in roots in five different sheets (Figure 3(b)).

It is well-known that just the first Riemann sheet has physical significance [49]. As such, in the sequel, we consider only the RL branches corresponding to the first sheet.

Observing the RL of $G_{1}(s)$, we verify that for $0<\alpha_{1}<1$, there are no closed-loop poles. However, for $1 \leq \alpha<4$, several graphs are obtained, as shown in Figure 4. Starting from the integer case $\left(\alpha_{1}=1\right)$ represented in Figure 4(a), as the FO pole increases, two branches emerge from the openloop pole $s=-2$ and flow towards infinity (Figure 4(b)). For $\alpha_{1}=2$, we get the classical plot with two vertical branches (Figure 4(c)). Increasing $\alpha_{1}\left(2<\alpha_{1}<3\right)$, two RL branches are still observed (Figure 4(d)). When $\alpha_{1}=3$, the wellknown three branches RL occurs (Figure 4(e)), and finally, when the FO pole is in the interval $3<\alpha_{1}<4$, four branches emerge. Larger values of the FO pole (i.e., $\alpha_{1} \geq 4$ ) were also investigated. We concluded that the RL sketching rules also apply. The results are of the same type, and therefore, we decided not to include them.

The practical rules apply to all FO cases. For example, for the RL shown in Figure 4(f), as $\alpha_{1}=3.5$, the RL has four branches. The asymptotes centroid and angles are $\sigma=-2$ and $\varphi=-154.3^{\circ},-51.4^{\circ}, 51.4^{\circ}$, and $154.3^{\circ}$, respectively. Solving the characteristic equation for $s=j \omega$, the RL branches intersect the imaginary axis at $\omega= \pm 2.51$, for $K=59.2$. 


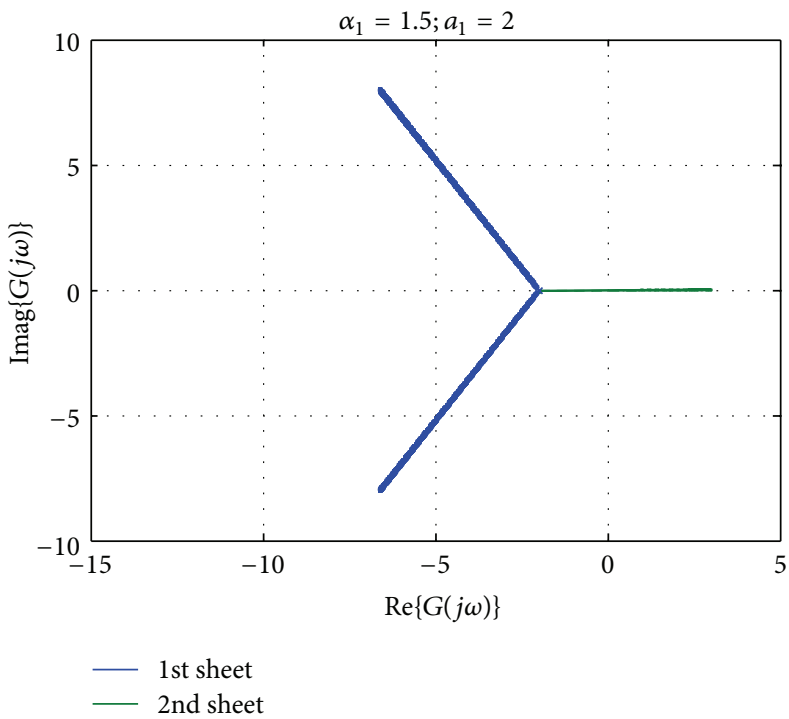

(a)

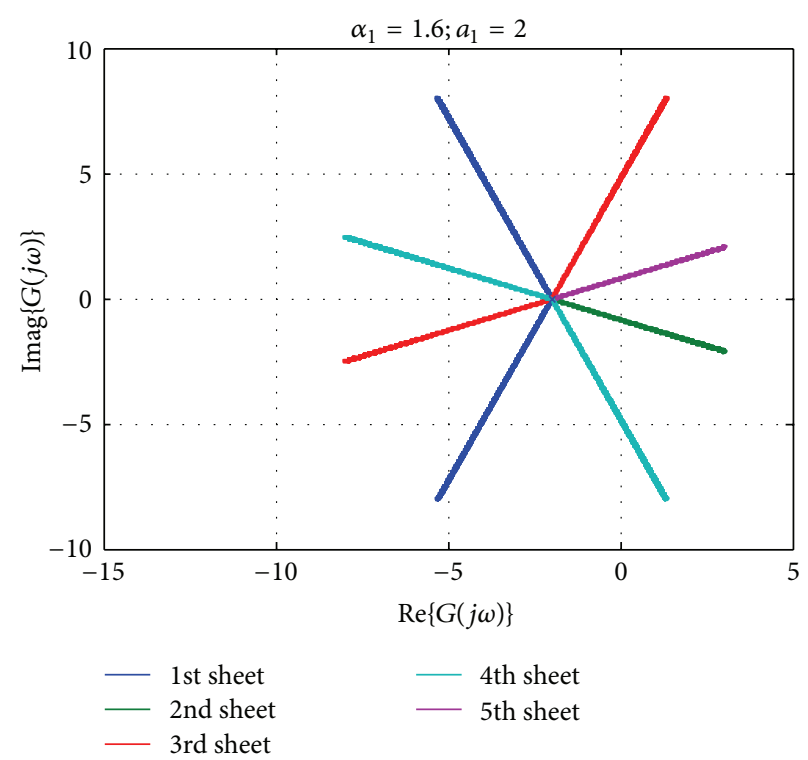

(b)

FIGURE 3: Root-Locus of $G_{1}(s)$ : (a) $a_{1}=2$ and $\alpha_{1}=1.5$ result in RL branches in two sheets; (b) $a_{1}=2$ and $\alpha_{1}=1.6$ result in RL branches in five sheets.

TABle 1: Practical rules for RL sketching of FO systems as defined in (11).

\begin{tabular}{|c|c|}
\hline & Fractional-order case, (11) \\
\hline Rule 1 & $\mathrm{RL}$ is symmetrical about the real axis. \\
\hline Rule 2 & $\begin{array}{l}\text { If } l<\sum_{p=1}^{n} \alpha_{p}<l+2, l=1,3,5, \ldots \\
\text { then the number of branches is } l+1\end{array}$ \\
\hline Rule 3 & Mark all open-loop poles and zeros on the s-plane. \\
\hline Rule 4 & If necessary, use the angle condition (1) to determine the open-loop poles that have RL branches departing from them. \\
\hline Rule 5 & $\begin{array}{l}\text { Compute the asymptotes centroid, } \sigma \text {, and angle, } \varphi \text {, according to the following expressions: } \\
\sigma=\frac{-\sum_{p=1}^{n} a_{p} \alpha_{p}+\sum_{q=1}^{m} b_{q} \beta_{q}}{\sum_{p=1}^{n} \alpha_{p}-\sum_{q=1}^{m} \beta_{q}} \\
\varphi=\frac{(2 h+1) \cdot 180^{\circ}}{\sum_{p=1}^{n} \alpha_{p}-\sum_{q=1}^{m} \beta_{q}}, h=0, \pm 1, \pm 2, \ldots\end{array}$ \\
\hline Rule 6 & $\begin{array}{l}\text { Points in the real axis belong to the RL if, for all poles and zeros seen to the right, } \delta=\sum_{p=1}^{n} \alpha_{p}-\sum_{q=1}^{m} \beta_{q} \text { is an integer odd } \\
\text { number. }\end{array}$ \\
\hline Rule 7 & $\begin{array}{l}\text { Find the intersection of the RL with the imaginary axis making } s=j \omega \text { in the characteristic equation and solving it in order to } \\
\text { determine } K \text { and } \omega \text {. }\end{array}$ \\
\hline Rule 8 & Compute the breakaway and break-in points using the characteristic equation and determining $d K / d s=0$ \\
\hline Rule 9 & Determine the departure and arrival angles using the angle condition (1). \\
\hline
\end{tabular}

3.2. Two Fractional-Order Real Poles. In this subsection, we consider the open-loop transfer function given by

$$
G_{2}(s)=\frac{K}{\left(s+a_{1}\right)^{\alpha_{1}}\left(s+a_{2}\right)^{\alpha_{2}}} .
$$

The RL was computed for various values of $\left\{\alpha_{1}, \alpha_{2}\right\}\left(a_{1}=\right.$ $\left.2, a_{2}=1\right)$ and the graphs analysed. It was observed that no RL branches exist when $\alpha_{12}=\alpha_{1}+\alpha_{2}<1$. Several RL examples are depicted in Figures 5 to 7 for $1 \leq \alpha_{12}<4$. The results are presented in three groups: (i) $1 \leq \alpha_{12}<2$; (ii) $2 \leq \alpha_{12}<3$; (iii) $3 \leq \alpha_{12}<4$. Similar results were observed for $\alpha_{12} \geq 4$ and $a_{1}<a_{2}$. For both cases, the practical sketching rules still apply.

Figure 5 shows the plots from group (i). When $\alpha_{12}=1$, the RL has a single branch in the real axis (Figures 5(a) and 5(b)). As $\alpha_{12}$ increases $\left(1<\alpha_{12}<2\right)$, two branches emerge from the poles $s=-2$ or $s=-1$, depending on the values of $\alpha_{1}$ and $\alpha_{2}$, and tend to infinity (Figures 5(c) to 5(f)).

As said in Section 3.1, all practical rules are valid for $G_{2}(s)$, (15). Using the case shown in Figure 5(f), for example, we have $\alpha_{1}+\alpha_{2}=1.9$, meaning that the RL has two branches. As we have two open-loop poles, rule 4 must be used to determine the pole from which the branches are departing. 


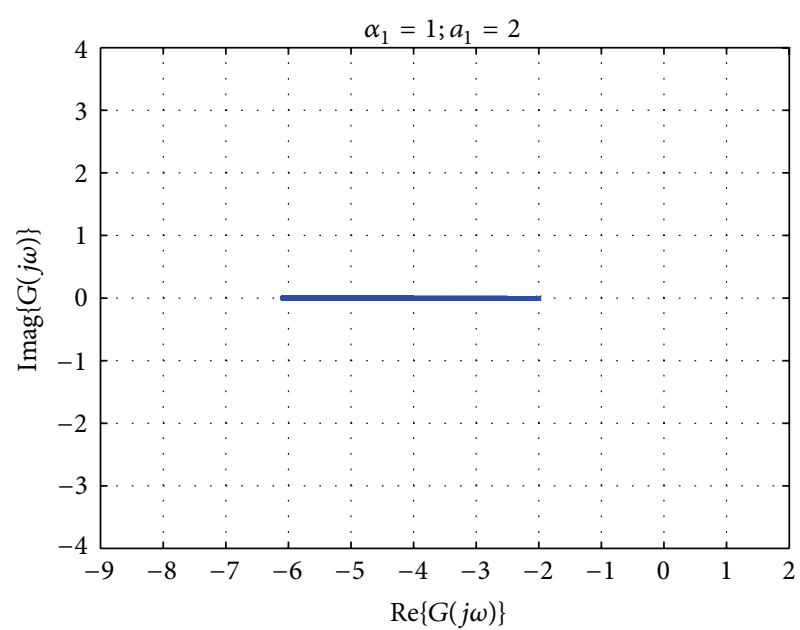

(a)

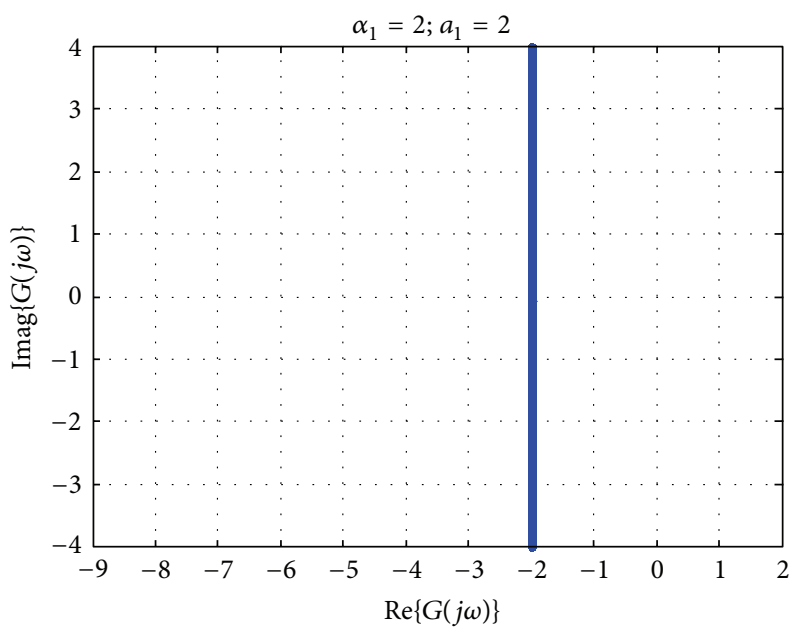

(c)

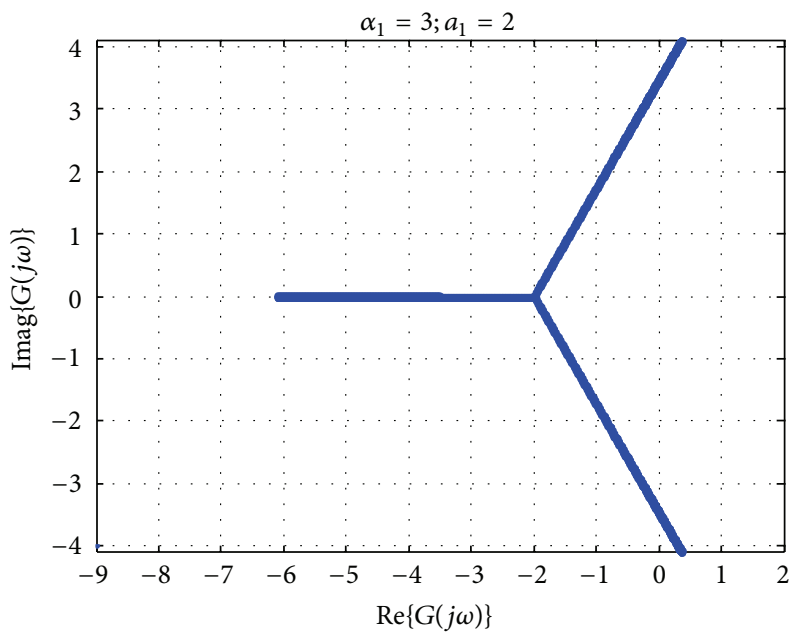

(e)

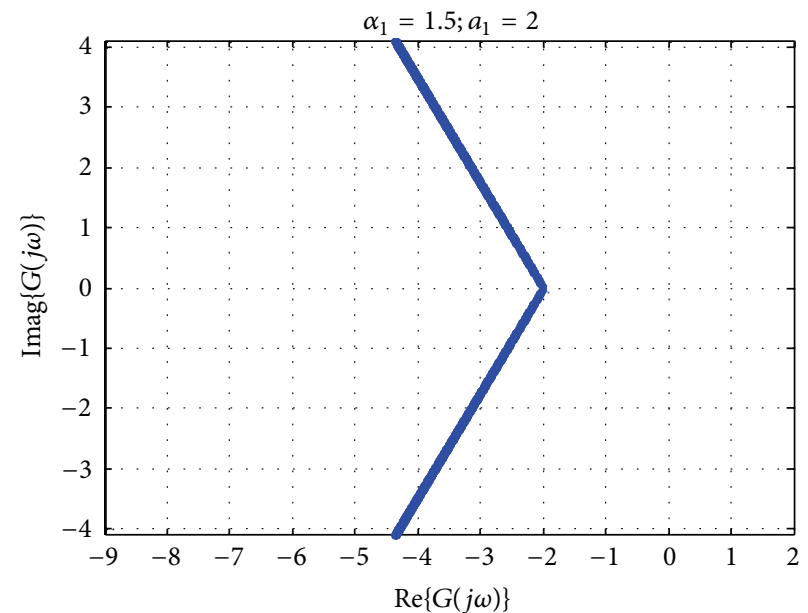

(b)

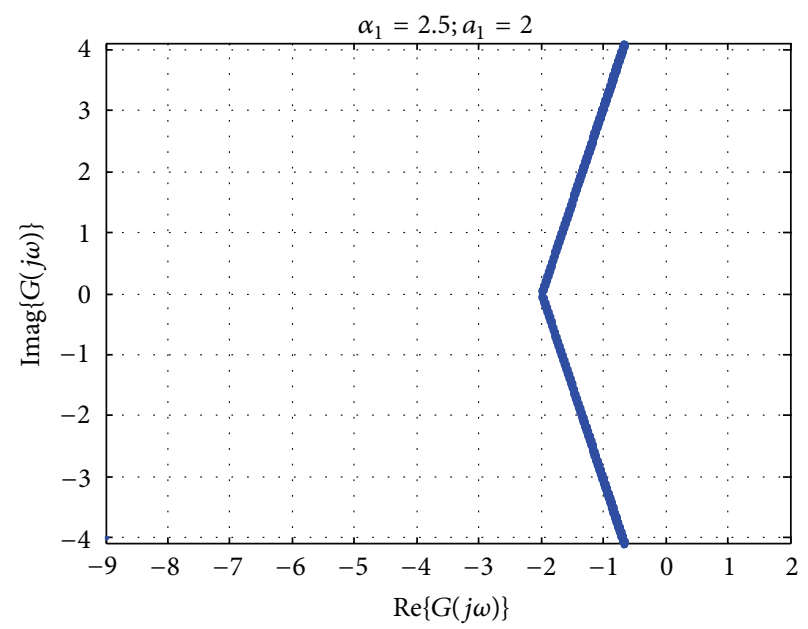

(d)

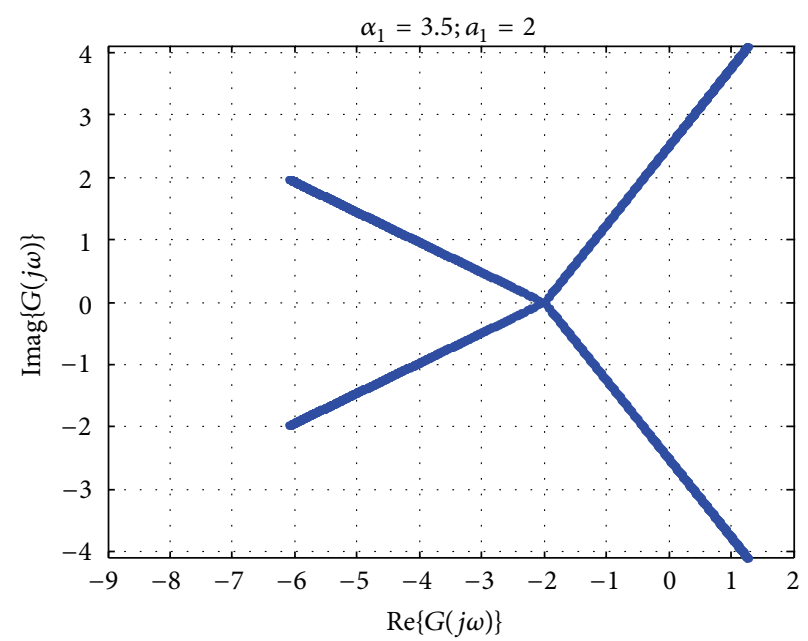

(f)

Figure 4: Root locus of $G_{1}(s)$ for $1 \leq \alpha_{1}<4$ and $a_{1}=2$. 


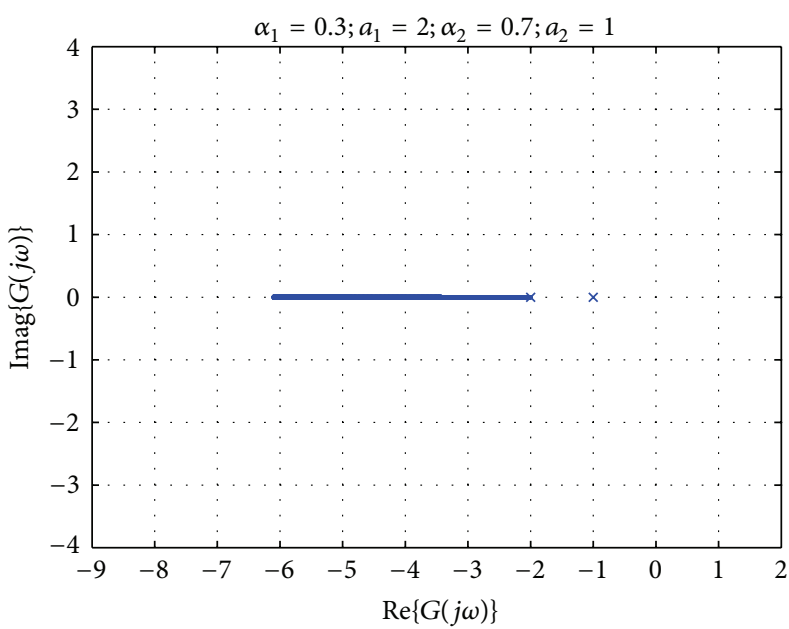

(a)

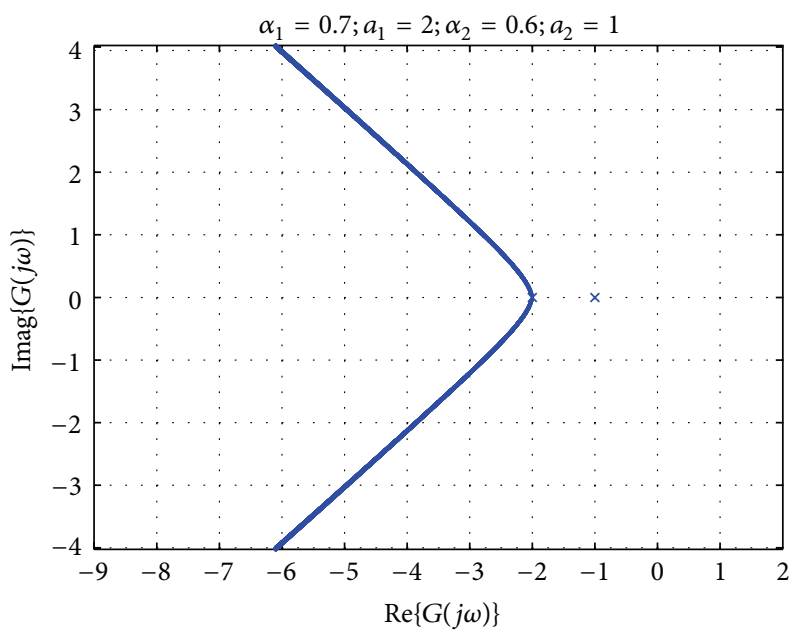

(c)

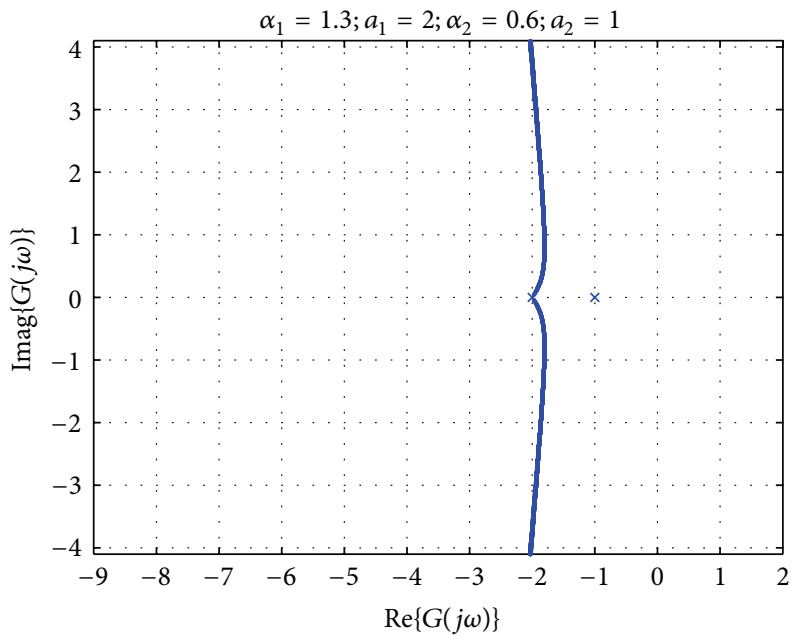

(e)

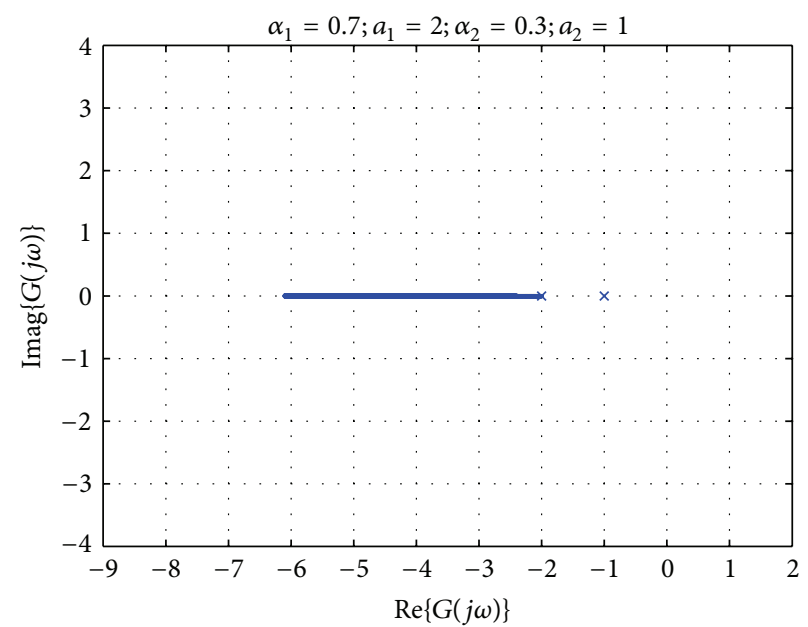

(b)

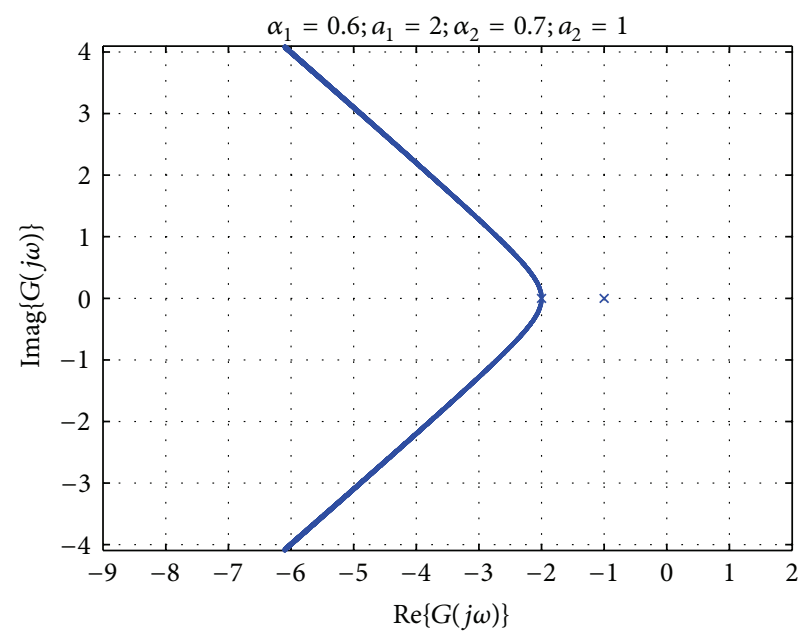

(d)

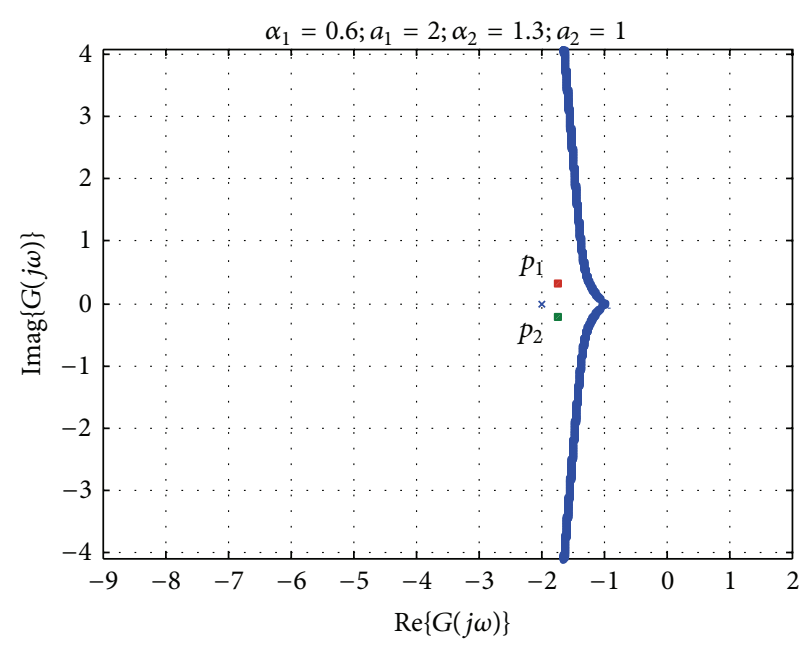

(f)

FIgURE 5: Root locus of $G_{2}(s)$. Cases from group (i), $1 \leq \alpha_{12}<2\left(a_{1}=2, a_{2}=1\right)$. 


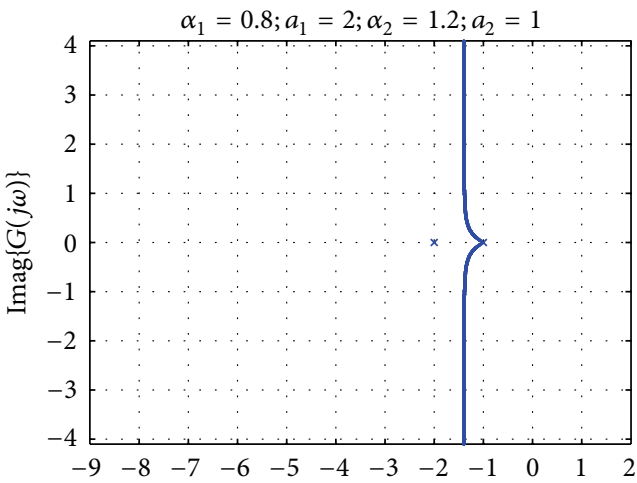

(a)

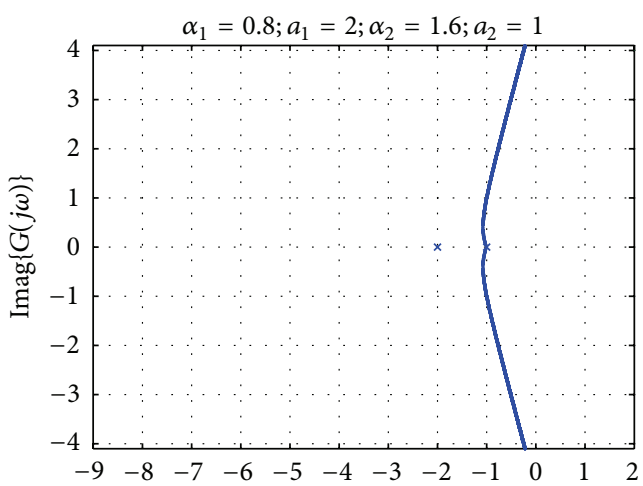

(c)

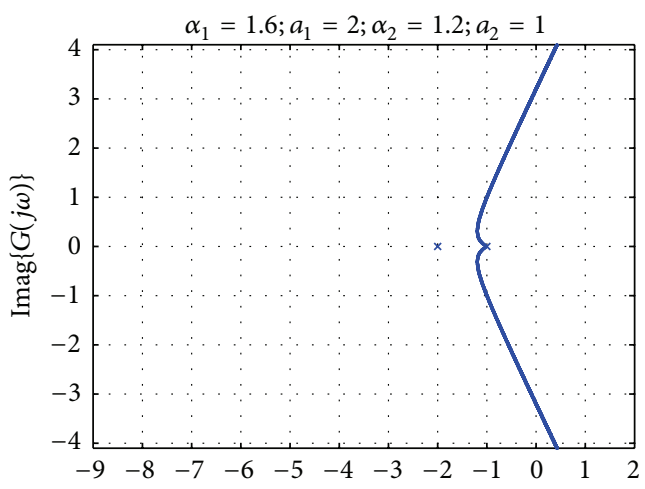

(e)

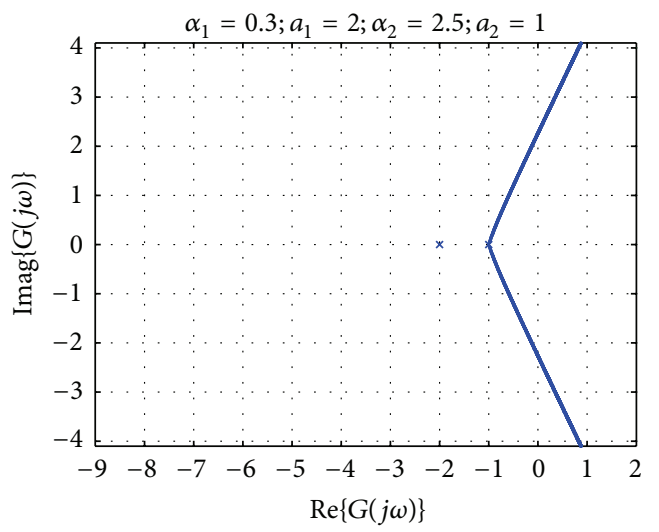

(g)

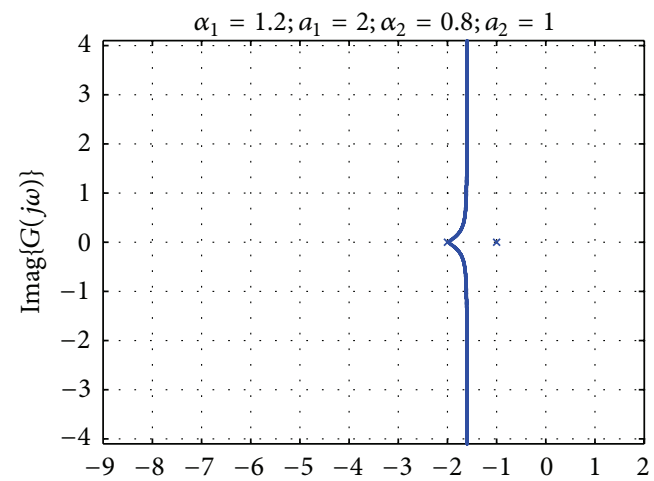

(b)

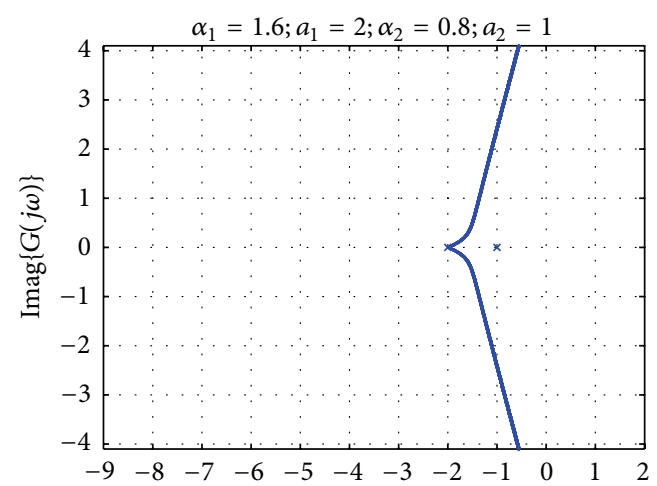

(d)

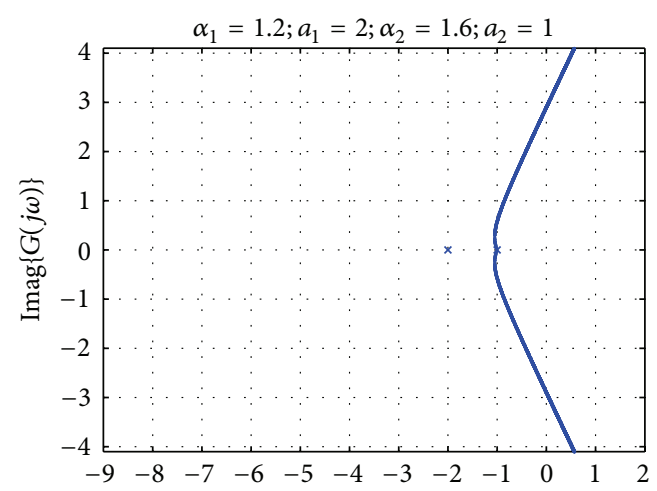

(f)

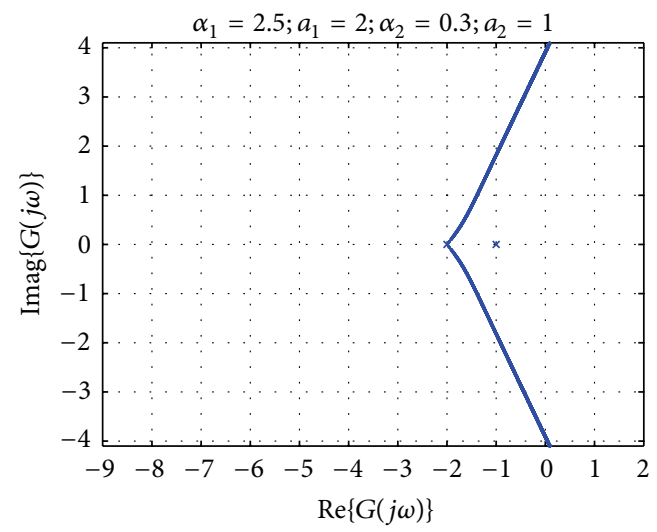

(h)

Figure 6: Root locus of $G_{2}(s)$. Cases from group (ii), $2 \leq \alpha_{12}<3\left(a_{1}=2, a_{2}=1\right)$. 


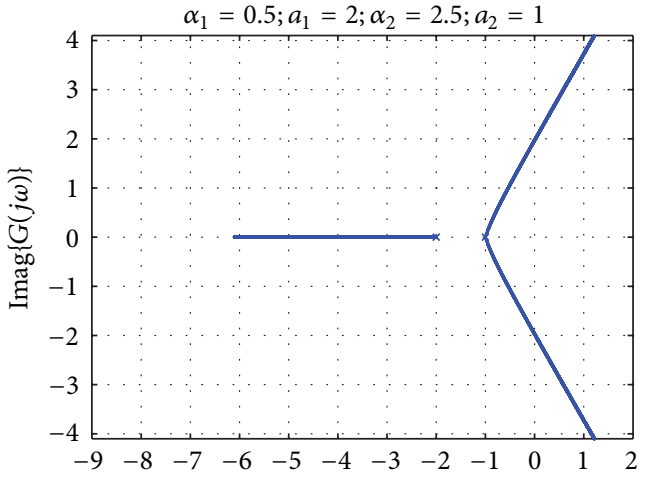

(a)

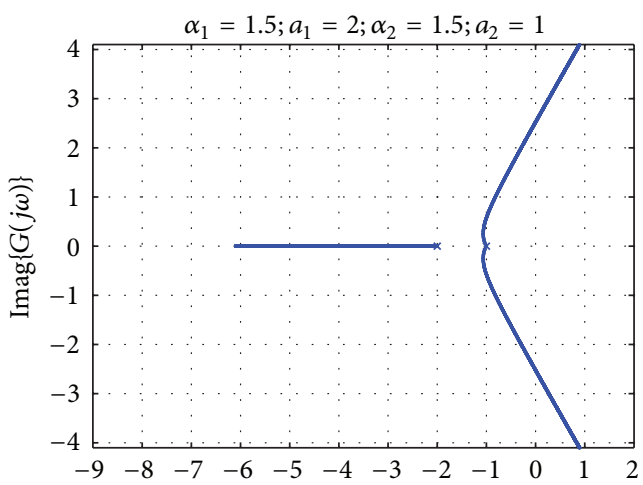

(c)

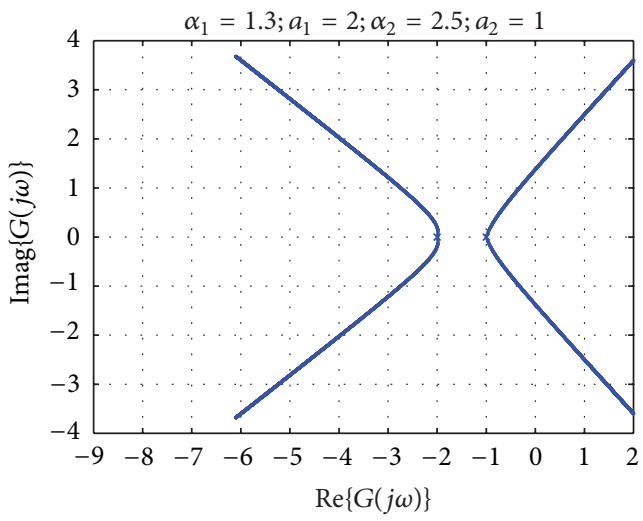

(e)

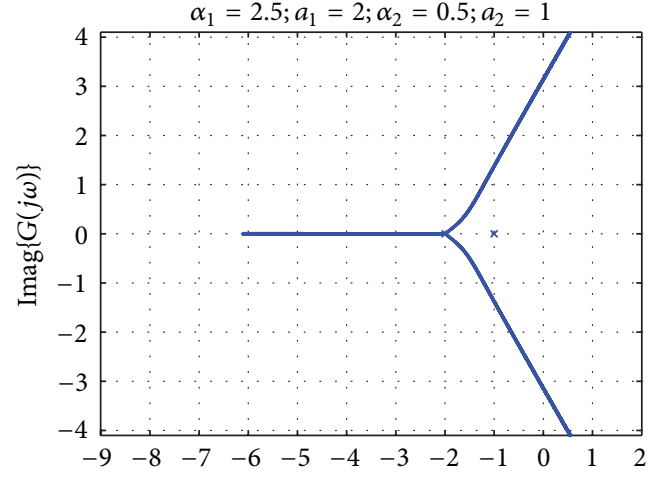

(b)

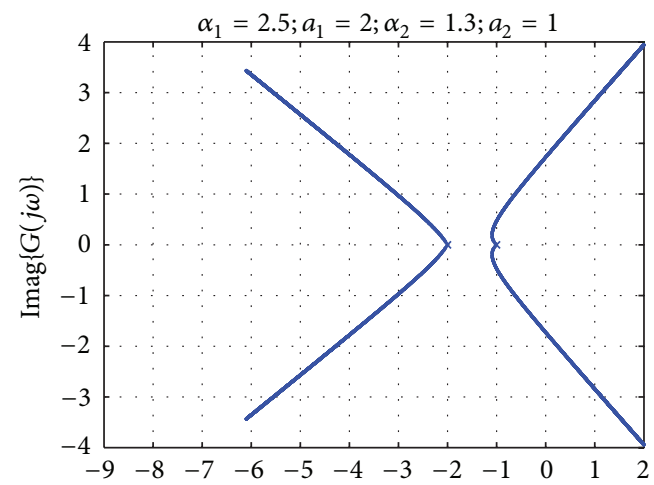

(d)

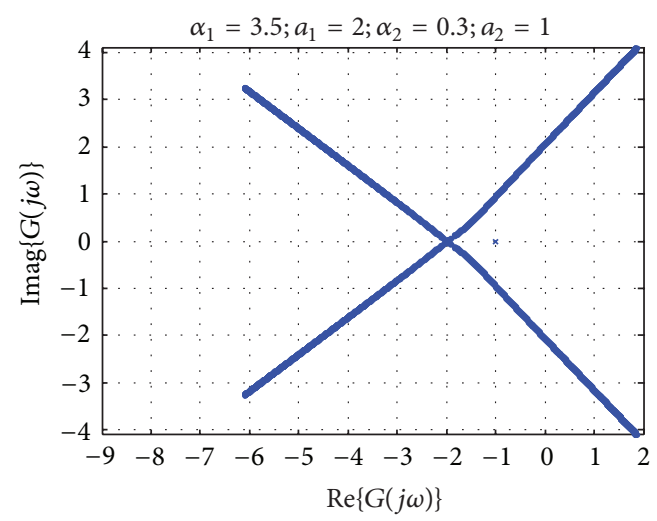

(f)

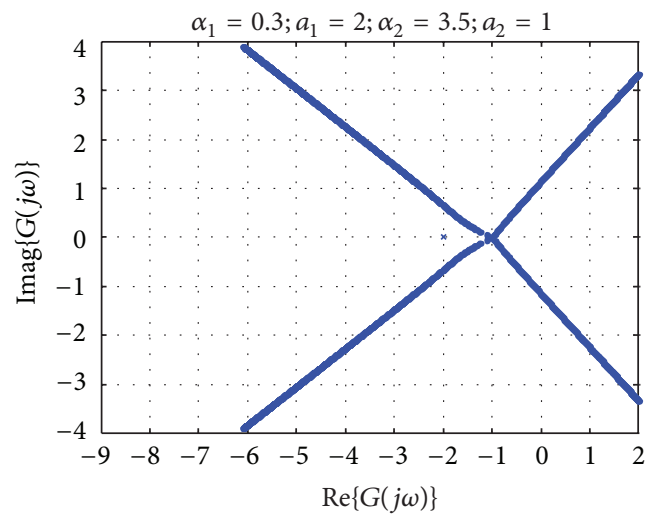

(g)

FIgURE 7: Root locus of $G_{2}(s)$. Cases from group (iii), $3 \leq \alpha_{12}<4\left(a_{1}=2, a_{2}=1\right)$. 


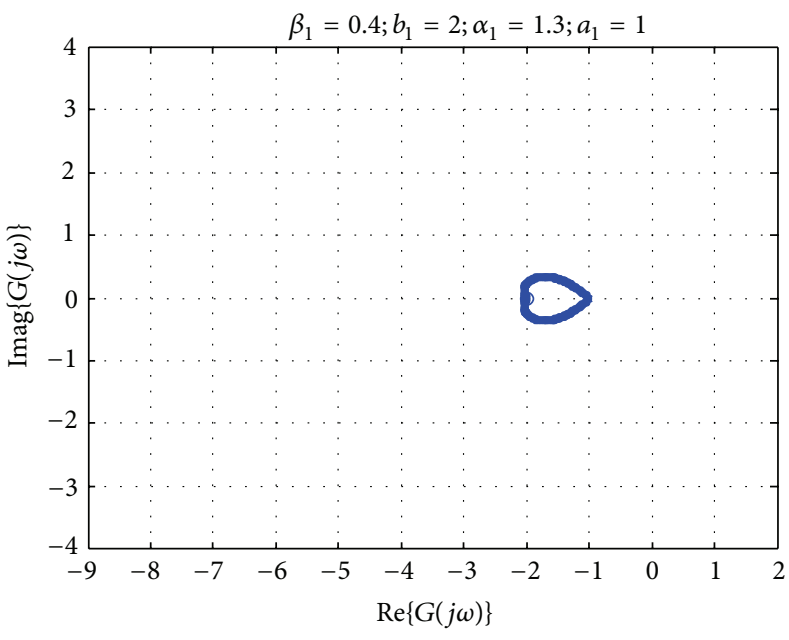

(a)

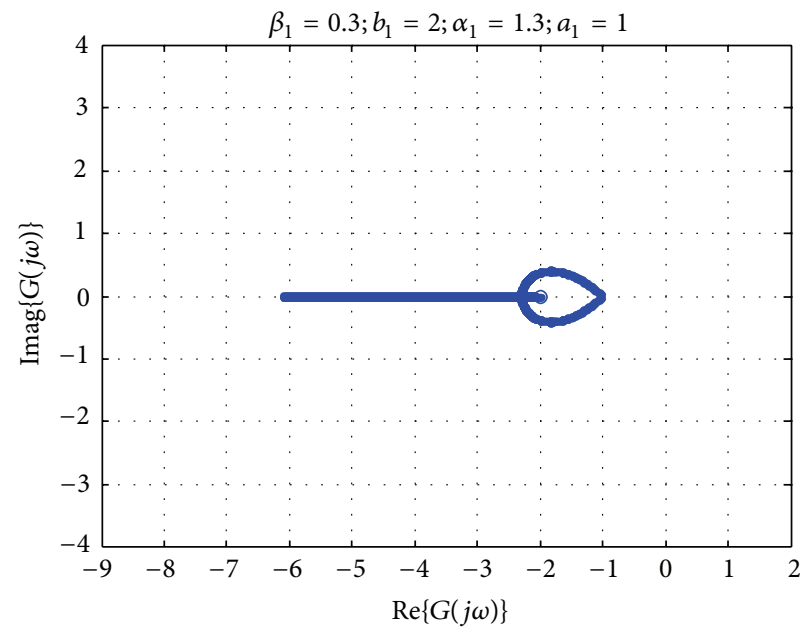

(b)

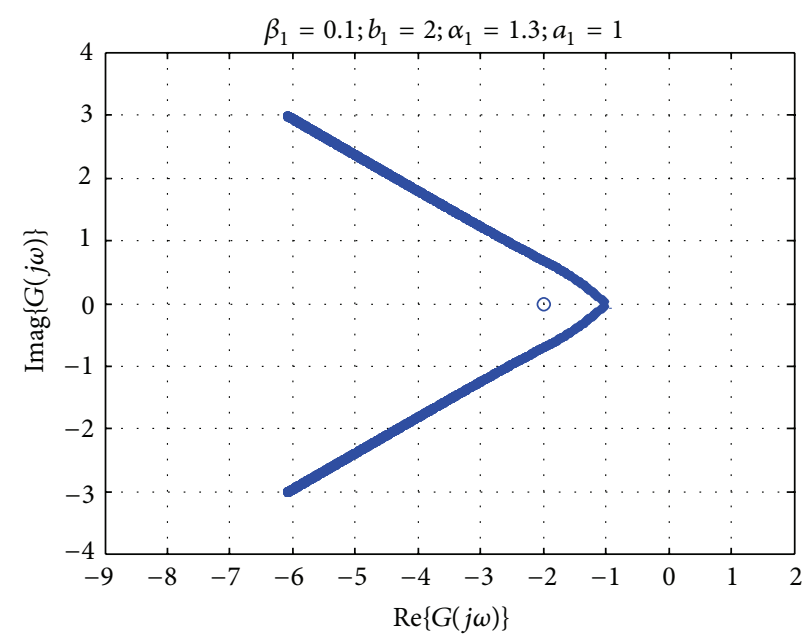

(c)

Figure 8: Root locus of $G_{3}(s)$. Cases from group (i), $1 \leq \alpha_{1}<2\left(a_{1}=1, b_{1}=2\right)$.

Thus, applying the angle condition to the test points $p_{1}$ and $p_{2}$, we obtain $\phi_{1}=-90^{\circ}$ and $\phi_{2}=90^{\circ}$, respectively, indicating that no branches can depart from $s=-2$. Rule 4 can be used in all cases; nevertheless, an easier to use specific rule about $\mathrm{RL}$ starting and ending points still requires more research before a definitive statement.

The angle condition is also used to determine the departing angles from pole $s=-1$, resulting in $\phi= \pm 138.46^{\circ}$. The asymptotes centroid and angles are $\sigma=-1.3$ and $\varphi= \pm 94.7^{\circ}$, respectively.

Figure 6 depicts results from group (ii). When $\alpha_{12}=2$, we get the plots represented in Figures 6(a) and 6(b). We observe two RL branches that, as before, depending on the values of $\alpha_{1}$ and $\alpha_{2}$, can depart from one or the other open-loop poles. In both cases, the branches tend to infinity with angles $\varphi= \pm 90^{\circ}$. Increasing the value of $\alpha_{12}\left(2<\alpha_{12}<3\right)$, two RL branches are still observed (Figures 6(c) to 6(h)).

The results from group (iii) are illustrated in Figure 7. For $\alpha_{12}=3$, the RL of Figures 7(a) to 7(c) shows three branches that depart from the same or different open-loop poles and flow to infinity with angles $\varphi=180^{\circ}$ and $\pm 60^{\circ}$. Increasing $\alpha_{12}$ $\left(3<\alpha_{12}<4\right)$, four RL branches arise (Figures $7(\mathrm{~d})$ to $7(\mathrm{~g})$ ).

The results obtained for two FO real poles are similar to those of a single real pole. This means a similar behavior, both in terms of the number of branches and the type of RL charts, whenever $\alpha_{1}$ and $\alpha_{12}$ are close. It should be noted that the RL depends not only on the equivalent order $\alpha_{12}$ (by means of rules 2,5 , or 6 ) but also on the FO of each pole. By other words, the same value of $\alpha_{12}$ may lead to different RL.

3.3. One Fractional-Order Pole and One Fractional-Order Zero. In this case, the open-loop transfer function is given by

$$
G_{3}(s)=\frac{K\left(s+b_{1}\right)^{\beta_{1}}}{\left(s+a_{1}\right)^{\alpha_{1}}} .
$$

The RL was obtained for various values of $\left\{\alpha_{1}, \beta_{1}\right\}\left(a_{1}=1\right.$, $\left.b_{1}=2\right)$ and the graphs analysed as previously. It was observed that no RL branches exist when $\alpha_{1}<1$. Figures 8 to 10 


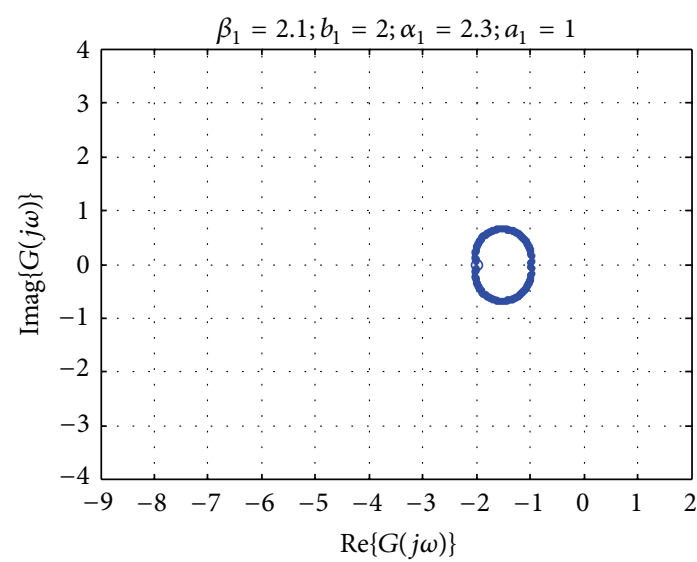

(a)

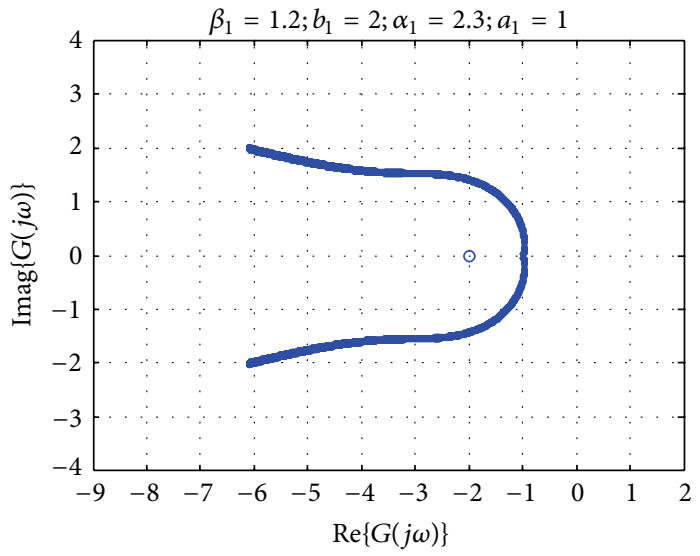

(c)

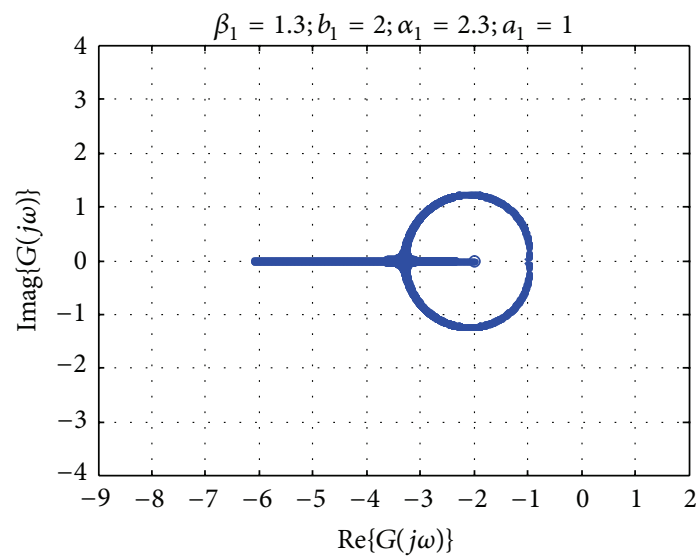

(b)

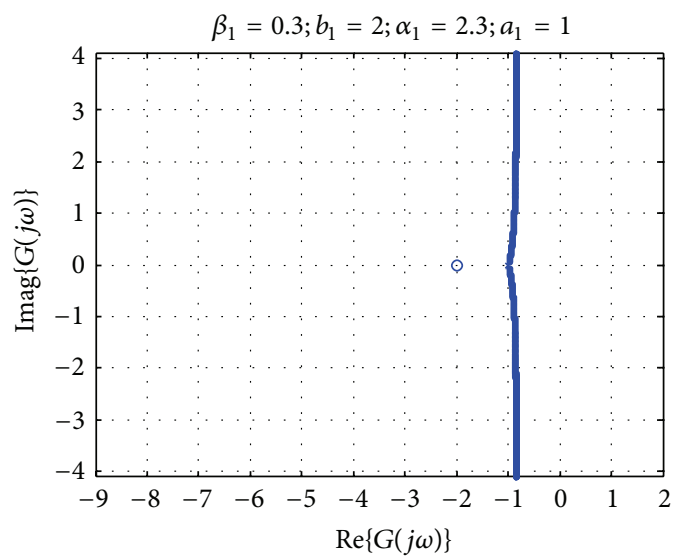

(d)

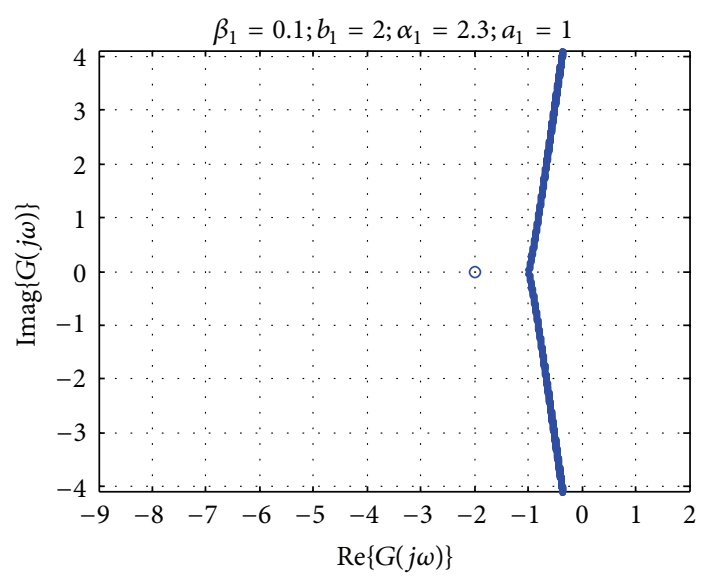

(e)

Figure 9: Root locus of $G_{3}(s)$. Cases from group (ii), $2 \leq \alpha_{1}<3\left(a_{1}=1, b_{1}=2\right)$.

depict several RL for $1 \leq \alpha_{1}<4$. As before, for easing the comparison, the results are presented in three groups: (i) $1 \leq \alpha_{1}<2$; (ii) $2 \leq \alpha_{1}<3$; (iii) $3 \leq \alpha_{1}<4$. Additional experiments were carried out, both for different values of the FO pole and FO zero and for $a_{1}>b_{1}$. We concluded that the sketching rules are valid for all cases and the results are similar to those presented.

Figure 8 shows plots from group (i). We see that each RL has two branches that depend on the difference between the orders of the denominator and numerator, $\delta=\alpha_{1}-\beta_{1}$ : 


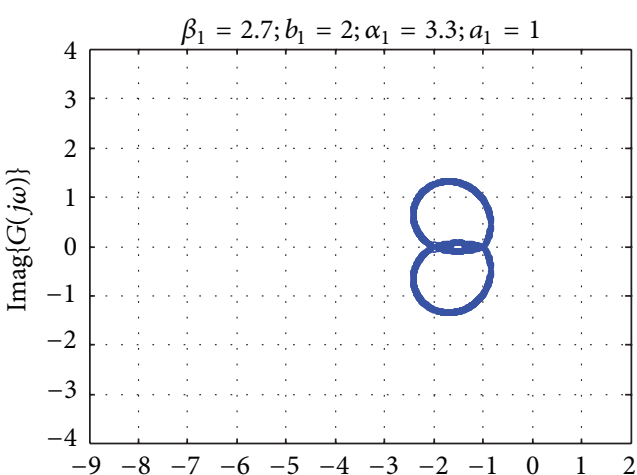

(a)

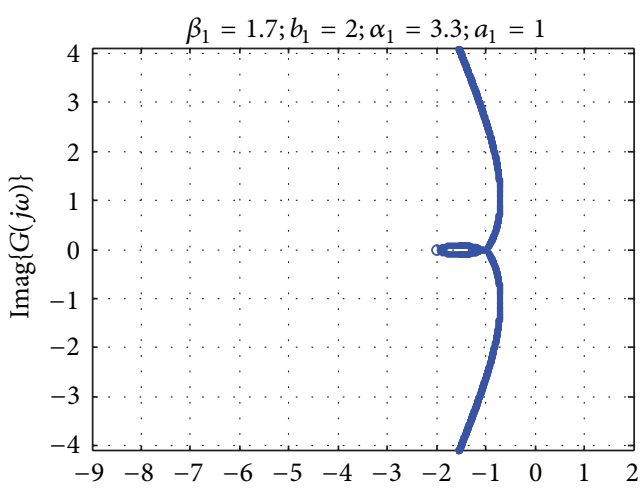

(c)

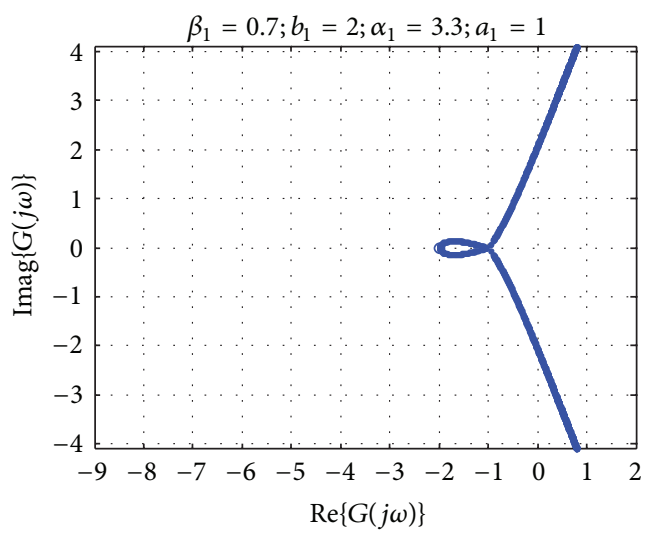

(e)

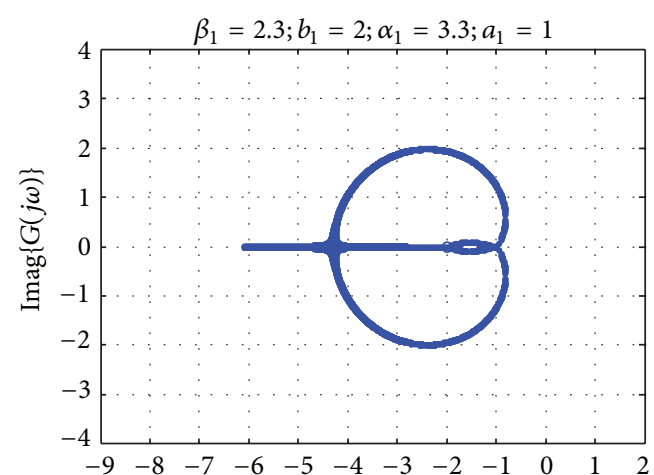

(b)

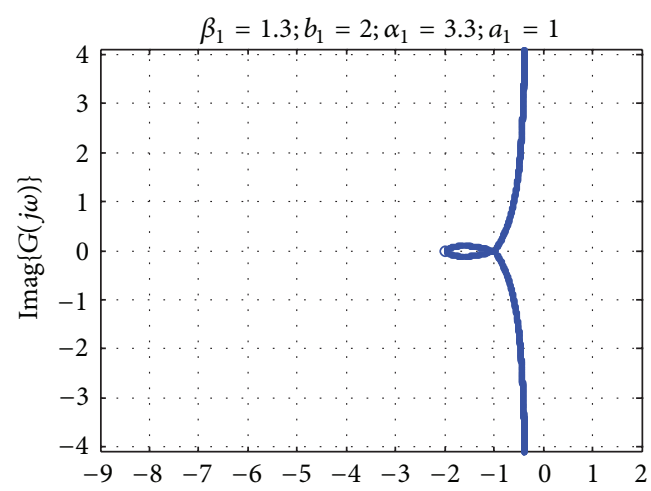

(d)

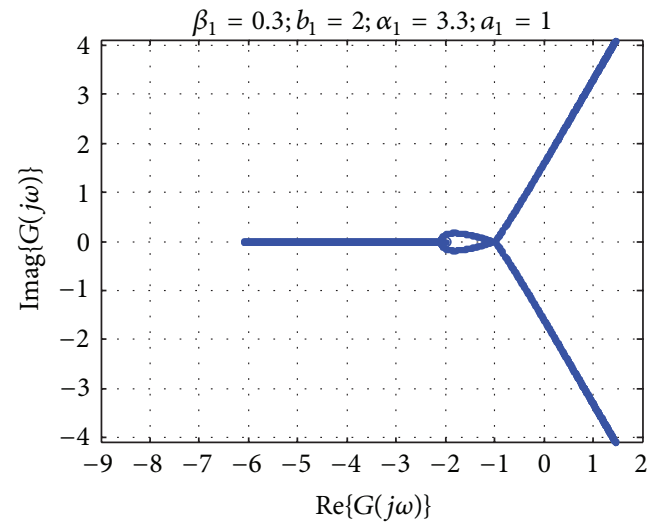

(f)

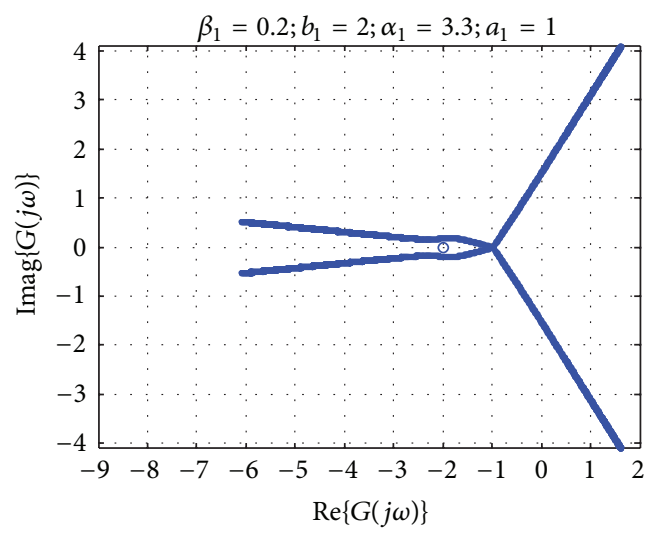

(g)

FIgURE 10: Root locus of $G_{3}(s)$. Cases from group (iii), $3 \leq \alpha_{1}<4\left(a_{1}=1, b_{1}=2\right)$. 


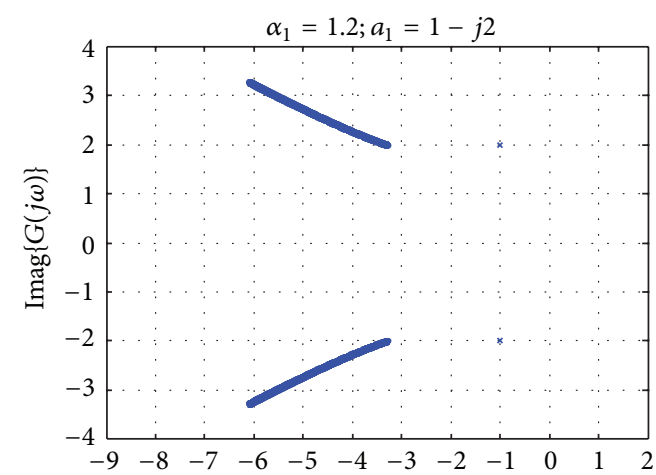

(a)

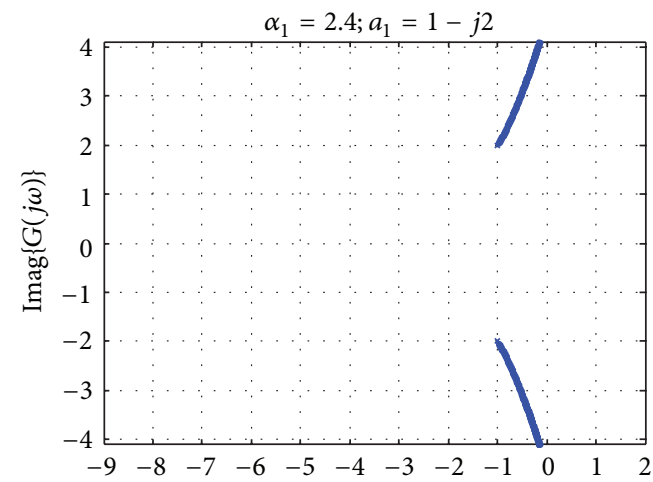

(c)

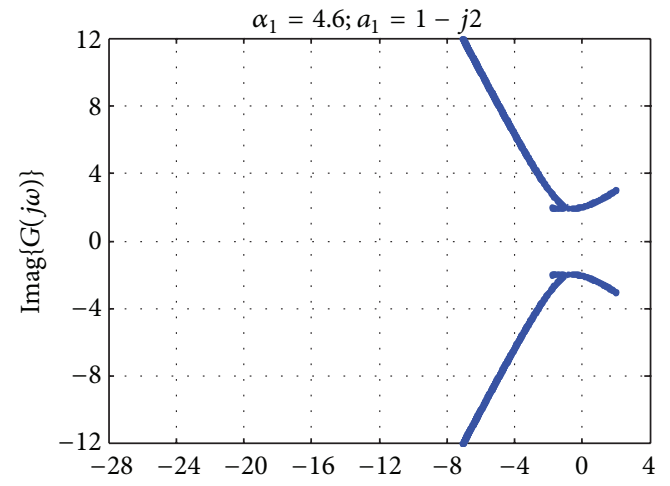

(e)

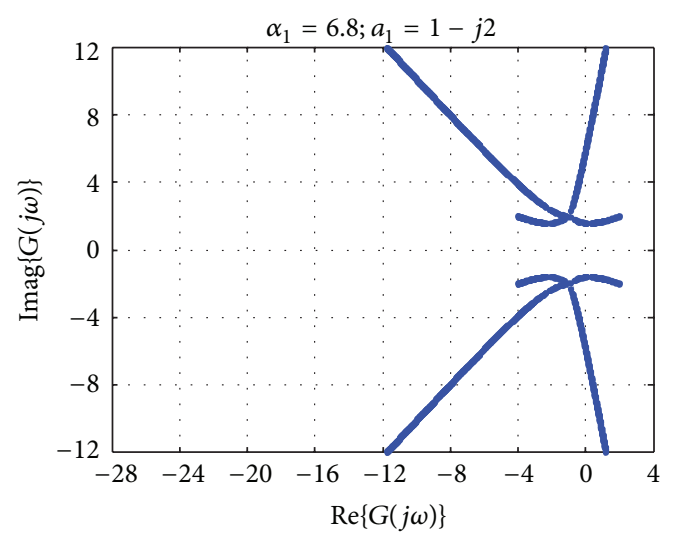

(g)

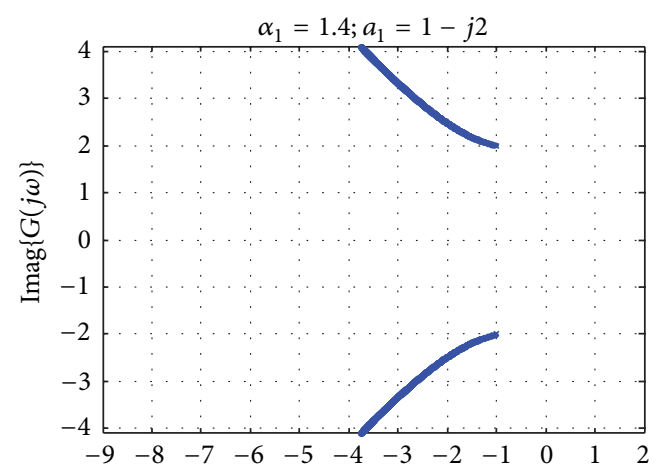

(b)

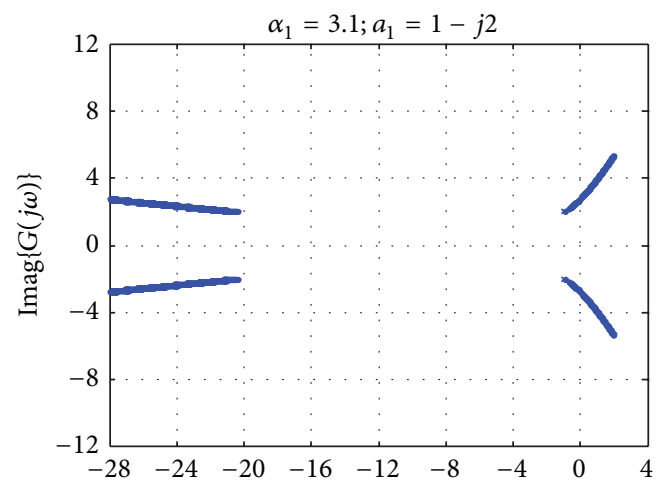

(d)

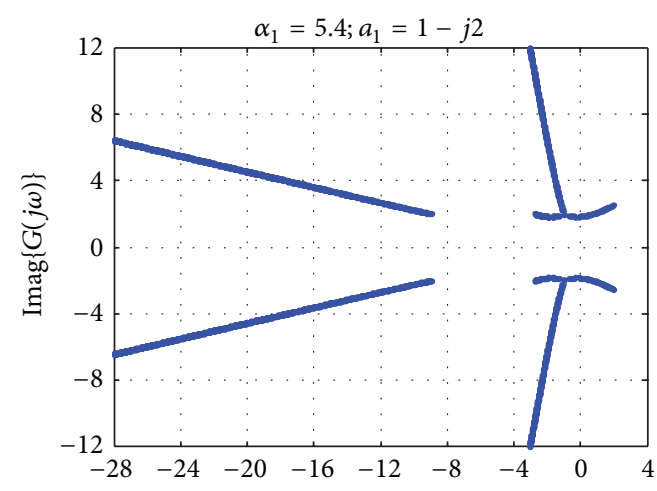

(f)

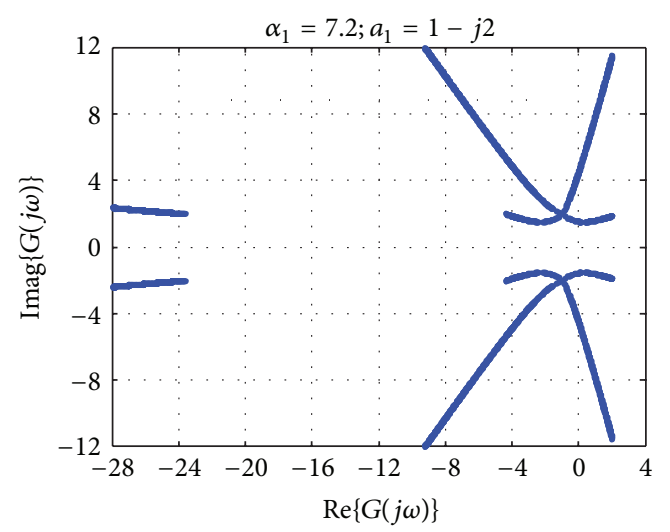

(h)

FIgURe 11: Root locus of $G_{4}(s)$ for $1<\alpha_{1}<8\left(a_{1}=1-j 2\right)$. 
when $\delta<1$, both branches converge to the open-loop zero (Figure 8(a)); if $\delta=1$, one branch converges to the openloop zero and the other tends to infinity (on the real axis) (Figure $8(\mathrm{~b})$ ); for $1<\delta<2$, the two branches flow to infinity (Figure 8(c)).

Applying rule 6 to the case depicted in Figure 8(b), for all real axis points in the line ] $-\infty,-2]$, we have $\delta=1$, meaning that this line belongs to the RL. The break-in point is computed using rule 8 , resulting in $s=-2.3$.

Figure 9 depicts several plots from group (ii), that is, $2 \leq$ $\alpha_{1}<3$. All RL still have two branches, the paths of which depend on the difference between the FO of the open-loop pole and zero (Figures 9(a) to 9(e)).

Several RL for group (iii), $3 \leq \alpha_{1}<4$, are shown in Figure 10. It can be observed that all RL have four branches, and as before, the paths depend on the difference between the orders of the open-loop pole and zero.

3.4. One Pair of Fractional-Order Complex Conjugate Poles. The open-loop transfer function is given by

$$
\begin{aligned}
G_{4}(s) & =\frac{K}{\left(s^{2}+2 \xi \omega_{n} s+\omega_{n}^{2}\right)^{\alpha_{1} / 2}} \\
& =\frac{K}{\left(s+a_{1}\right)^{\alpha_{1} / 2} \cdot\left(s+a_{1}^{*}\right)^{\alpha_{1} / 2}},
\end{aligned}
$$

where $a_{1} \in \mathrm{C}$ and $a_{1}^{*}$ denotes the conjugate of $a_{1}$.

Plotting the RL, it can be seen that there are no branches unless $\alpha_{1} \geq 1$. In Figure 11, several RL graphs are shown for $1<\alpha_{1}<8$. Figure 11(a) depicts the RL for $\alpha_{1}=1.2$, where we can see that there are gaps between the open-loop poles and the points were the branches initiate. Recalling that the RL can spread along several Riemann sheets, meaning that RL branches can begin in one sheet, cross the branch cut, and enter in another sheet, the gaps correspond to points not belonging to the first Riemann sheet. As in the previous examples, when $1<\alpha_{1}<3$, the RL has two branches (Figures 11(a) to 11(c)). When $3<\alpha_{1}<5$, the number of branches is four. Even though, for $3<\alpha_{1}<4$, there are gaps in two branches (Figure 11(d)), and for $4<\alpha_{1}<5$, two extra small branches depart from the open-loop poles and end close to those points, entering in another Riemann sheet (Figure 11(e)). The same qualitative behaviour is observed for $5<\alpha_{1}<7$ (Figures 11(f) to 11(g)). Figure 11(h) depicts the RL for $7<\alpha_{1}<8$, revealing eight branches departing from the open-loop poles.

To conclude the analysis, we use the case shown in Figure $11(\mathrm{~g})$ to underline that all $\mathrm{RL}$ practical rules are applicable, namely, the asymptotes centroid and angles, which are $\sigma=-1$ and $\varphi=-158.8^{\circ},-52.9^{\circ}, 52.9$, and $158.8^{\circ}$, respectively. The angle condition is used to determine the departing angles from pole $s=-1+j 2$, resulting in the values $\phi=-142.9,-37.1^{\circ}, 68.8$, and $174.7^{\circ}$.

\section{Conclusion}

The Root-Locus (RL) is a classical method for the analysis and synthesis of linear time-invariant (LTI) integer-order systems, consisting of the plot of the paths of all possible closed-loop poles as a design parameter varies in a given range. Nowadays, there are efficient numerical algorithms devoted to RL analysis, implemented by several packages. For integer-order systems, there are well-known practical rules for RL sketching, but those cannot be directly applied to FO systems, and the existing literature on this topic almost exclusively focuses on particular cases, namely, the commensurate FO systems.

This paper generalises RL practical rules to a class of FO systems, which are defined by an open-loop transfer function expressed as a ratio of FO zeros and poles. As usual, using practical rules, even though the RL sketch might result somewhat incomplete, the ability to quickly sketch RL by hand is invaluable, from the control designer viewpoint, in making fundamental decisions early in the design process.

\section{References}

[1] W. R. Evans, "Graphical analysis of control systems," Transactions of the American Institute of Electrical Engineers, vol. 67, no. 1, pp. 547-551, 1948.

[2] W. R. Evans, "Control systems synthesis by root locus method," Transactions of the American Institute of Electrical Engineers, vol. 69, no. 1, pp. 66-69, 1950.

[3] A. M. Krall, "The root locus method: a survey," SIAM Review, vol. 12, pp. 64-72, 1970.

[4] A. M. Eydgahi and M. Ghavamzadeh, "Complementary root locus revisited," IEEE Transactions on Education, vol. 44, no. 2, pp. 137-143, 2001.

[5] E. Bahar and M. Fitzwater, "Numerical technique to trace the loci of the complex roots of characteristic equations," SIAM Journal on Scientific and Statistical Computing, vol. 2, no. 4, pp. 389-403, 1981.

[6] C. I. Byrnes, D. S. Gilliam, and J. He, "Root-locus and boundary feedback design for a class of distributed parameter systems," SIAM Journal on Control and Optimization, vol. 32, no. 5, pp. 1364-1427, 1994.

[7] http://www.mathworks.com/.

[8] http://www.gnu.org/software/octave/.

[9] http://www.scilab.org/.

[10] http://freemat.sourceforge.net/.

[11] D. Matignon, "Stability properties for generalized fractional differential systems," in Systèmes Différentiels Fractionnaires, vol. 5, pp. 145-158, 1998.

[12] D. Matignon, "Stability results on fractional differential equations with applications to control processing," in Proceedings of the Computational Engineering in Systems Applications (CESA '96) IMACSIEEE/SMC Multiconference, pp. 963-968, Lille, France, 1996.

[13] C. F. Lorenzo and T. T. Hartley, "Initialization, conceptualization, and application in the generalized (Fractional) calculus," NASA TP-1998-208415, 1998.

[14] I. Petráš, "Stability of fractional-order systems with rational orders: a survey," Fractional Calculus \& Applied Analysis, vol. 12, no. 3, pp. 269-298, 2009.

[15] F. Merrikh-Bayat, M. Afshar, and M. Karimi-Ghartemani, "Extension of the root-locus method to a certain class of fractional-order systems," ISA Transactions, vol. 48, no. 1, pp. 48-53, 2009. 
[16] F. Merrikh-Bayat and M. Afshar, "Extending the root-locus method to fractional-order systems," Journal of Applied Mathematics, vol. 2008, Article ID 528934, 13 pages, 2008.

[17] J. A. T. Machado, "Root locus of fractional linear systems," Communications in Nonlinear Science and Numerical Simulation, vol. 16, no. 10, pp. 3855-3862, 2011.

[18] J. A. T. Machado, "A gallery of root locus of fractional systems," in Proceedings of the ASME International Design Engineering Technical Conferences \& Computers and Information in Engineering Conference (DETC '13), Portland, Ore, USA, 2013.

[19] S. G. Samko, A. A. Kilbas, and O. I. Marichev, Fractional Integrals and Derivatives, Gordon and Breach Science Publishers, London, UK, 1993.

[20] K. S. Miller and B. Ross, An Introduction to the Fractional Calculus and Fractional Differential Equations, John Wiley \& Sons, New York, NY, USA, 1993.

[21] I. Podlubny, Fractional Differential Equations, vol. 198, Academic Press, San Diego, CA, USA, 1999.

[22] A. A. Kilbas, H. M. Srivastava, and J. J. Trujillo, Theory and Applications of Fractional Differential Equations, North-Holland Mathematics Studies, Elsevier, Amsterdam, The Netherlands, 2006.

[23] K. Diethelm, The Analysis of Fractional Differential Equations, Springer, Berlin, Germany, 2010.

[24] A. Oustaloup, La Commande CRONE: Commande Robuste D’Ordre Non Entier, Hermès, Paris, France, 1991.

[25] G. M. Zaslavsky, Hamiltonian Chaos and Fractional Dynamics, Oxford University Press, New York, NY, USA, 2008.

[26] R. Magin, Fractional Calculus in Bioengineering, Begell House Publishers, Redding, CA, USA, 2006.

[27] F. Mainardi, Fractional Calculus and Waves in Linear Viscoelasticity, Imperial College Press, London, UK, 2010.

[28] C. A. Monje, Y. Chen, B. M. Vinagre, D. Xue, and V. Feliu, Fractional Order Systems and Controls: Fundamentals and Applications, Springer, London, UK, 2010.

[29] J. T. Machado, V. Kiryakova, and F. Mainardi, "Recent history of fractional calculus," Communications in Nonlinear Science and Numerical Simulation, vol. 16, no. 3, pp. 1140-1153, 2011.

[30] T. J. Anastasio, "The fractional-order dynamics of brainstem vestibulo-oculomotor neurons," Biological Cybernetics, vol. 72, no. 1, pp. 69-79, 1994.

[31] J.-G. Lu and Y.-Q. Chen, "Robust stability and stabilization of fractional-order interval systems with the fractional order alpha: the $0<\alpha<1$ Case," IEEE Transactions on Automatic Control, vol. 55, no. 1, pp. 152-158, 2010.

[32] D. Baleanu, A. K. Golmankhaneh, A. K. Golmankhaneh, and R. R. Nigmatullin, "Newtonian law with memory" Nonlinear Dynamics, vol. 60, no. 1-2, pp. 81-86, 2010.

[33] C. M. Ionescu, J. A. T. Machado, and R. de Keyser, "Modeling of the lung impedance using a fractional-order ladder network with constant phase elements," IEEE Transactions on Biomedical Circuits and Systems, vol. 5, no. 1, pp. 83-89, 2011.

[34] E. Scalas, R. Gorenflo, and F. Mainardi, "Fractional calculus and continuous-time finance," Physica A, vol. 284, no. 1-4, pp. 376384, 2000.

[35] F. B. Duarte, J. A. T. Machado, and G. Monteiro Duarte, "Dynamics of the Dow Jones and the NASDAQ stock indexes," Nonlinear Dynamics, vol. 61, no. 4, pp. 691-705, 2010.

[36] C. M. Ionescu, P. Segers, and R. de Keyser, "Mechanical properties of the respiratory system derived from morphologic insight," IEEE Transactions on Biomedical Engineering, vol. 56, no. 4, pp. 949-959, 2009.

[37] C. Ionescu and J. T. Machado, "Mechanical properties and impedance model for the branching network of the sapping system in the leaf of Hydrangea Macrophylla," Nonlinear Dynamics, vol. 60, no. 1-2, pp. 207-216, 2010.

[38] J. A. T. Machado and A. M. Lopes, "Dynamical analysis of the global warming," Mathematical Problems in Engineering, vol. 2012, Article ID 971641, 12 pages, 2012.

[39] J. A. T. Machado, A. C. Costa, and M. D. Quelhas, "Fractional dynamics in DNA," Communications in Nonlinear Science and Numerical Simulation, vol. 16, no. 8, pp. 2963-2969, 2011.

[40] W. H. Deng and C. P. Li, "Chaos synchronization of the fractional Lü system,” Physica A, vol. 353, no. 1-4, pp. 61-72, 2005.

[41] R. R. Nigmatullin, “'Fractional' kinetic equations and 'universal' decoupling of a memory function in mesoscale region," Physica A, vol. 363, no. 2, pp. 282-298, 2006.

[42] O. P. Agrawal, "Fractional variational calculus in terms of Riesz fractional derivatives," Journal of Physics A, vol. 40, no. 24, pp. 6287-6303, 2007.

[43] A. Oustaloup, X. Moreau, and M. Nouillant, "The crone suspension," Control Engineering Practice, vol. 4, no. 8, pp. 1101-1108, 1996.

[44] A. G. Radwan, A. M. Soliman, and A. S. Elwakil, "Design equations for fractional-order sinusoidal oscillators: four practical circuit examples," International Journal of Circuit Theory and Applications, vol. 36, no. 4, pp. 473-492, 2008.

[45] I. Petráš, "A note on the fractional-order Chua's system," Chaos, Solitons and Fractals, vol. 38, no. 1, pp. 140-147, 2008.

[46] H. L. Cao, Z. H. Deng, X. Li, J. Yang, and Y. Qin, "Dynamic modeling of electrical characteristics of solid oxide fuel cells using fractional derivatives," International Journal of Hydrogen Energy, vol. 35, no. 4, pp. 1749-1758, 2010.

[47] J. A. T. Machado, "Fractional order modelling of fractionalorder holds," Nonlinear Dynamics, vol. 70, no. 1, pp. 789-796, 2012.

[48] I. Petras, Fractional-Order Nonlinear Systems: Modeling, Analysis and Simulation, Springer, New York, NY, USA, 2011.

[49] B. Gross and E. P. Braga, Singularities of Linear System Functions, Elsevier, New York, NY, USA, 1961. 


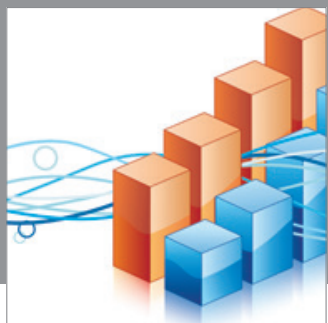

Advances in

Operations Research

mansans

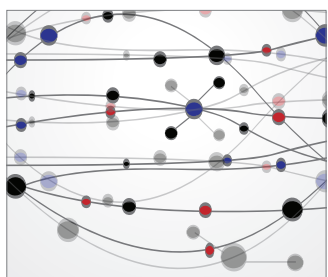

The Scientific World Journal
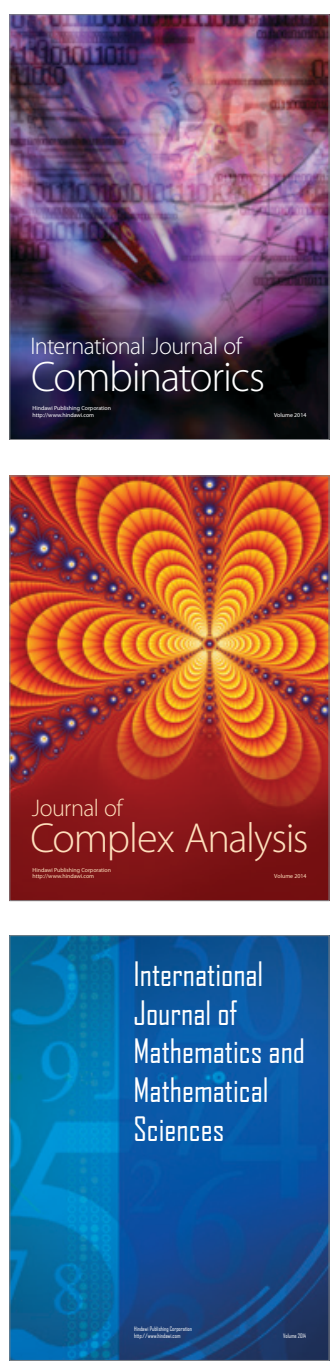
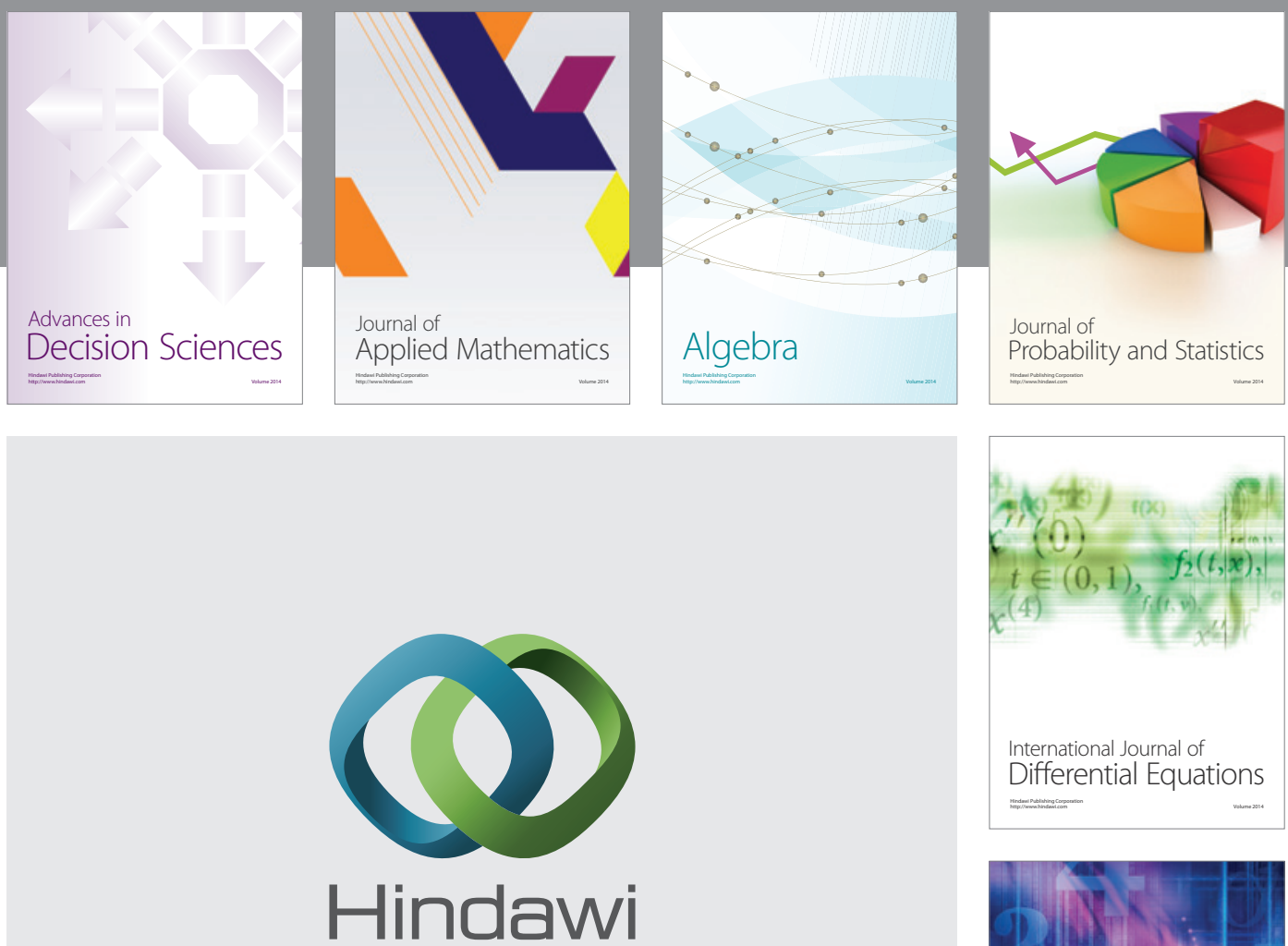

Submit your manuscripts at http://www.hindawi.com
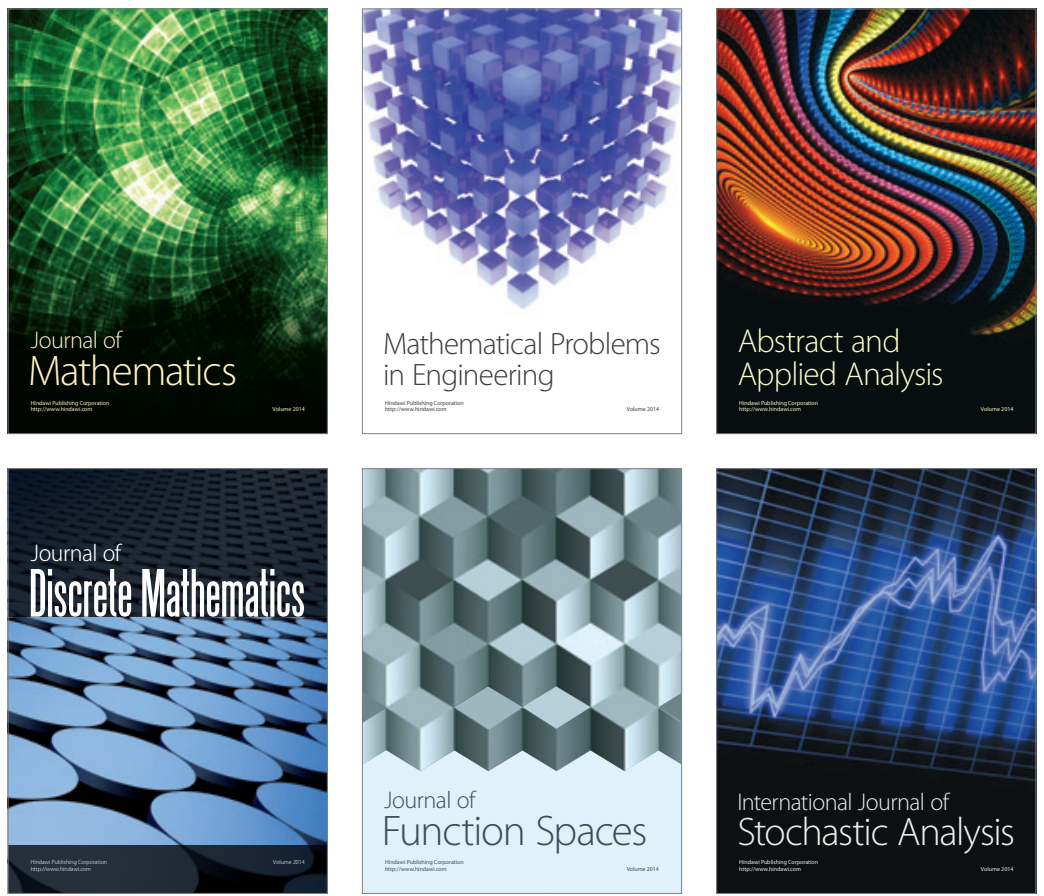

Journal of

Function Spaces

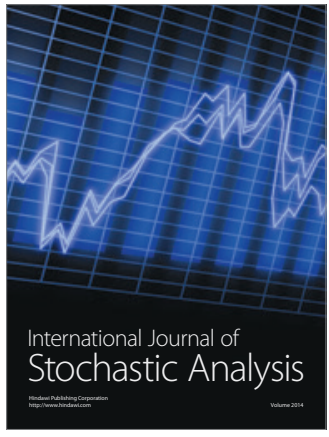

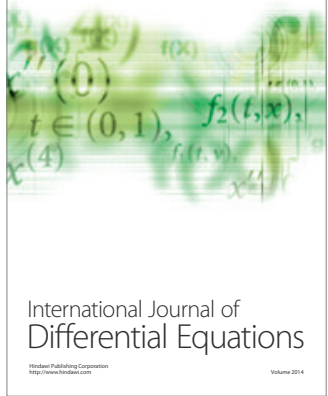
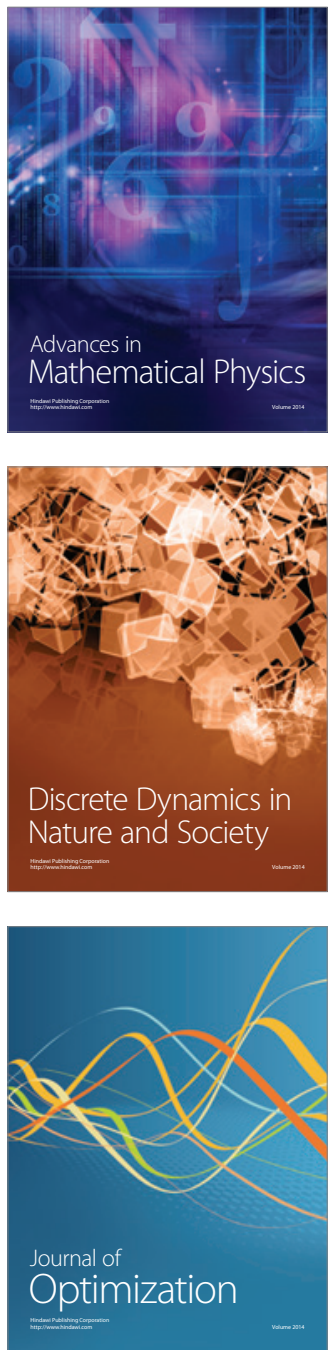